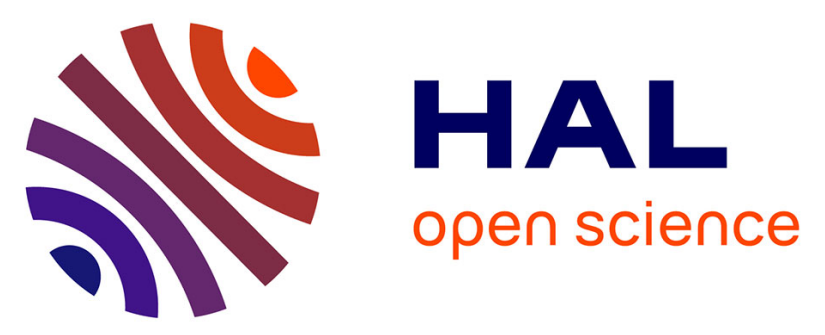

\title{
Self-induced transparency scenario revisited via beat-wave heating induced by Doppler shift in overdense plasma layer
}

\author{
A. Ghizzo, D. del Sarto, T. Reveille, Nicolas Besse, R. Klein
}

\section{- To cite this version:}

A. Ghizzo, D. del Sarto, T. Reveille, Nicolas Besse, R. Klein. Self-induced transparency scenario revisited via beat-wave heating induced by Doppler shift in overdense plasma layer. Physics of Plasmas, 2007, 14 (6), pp.062702. 10.1063/1.2735123 . hal-01804607

\section{HAL Id: hal-01804607 \\ https://hal.univ-lorraine.fr/hal-01804607}

Submitted on 31 May 2018

HAL is a multi-disciplinary open access archive for the deposit and dissemination of scientific research documents, whether they are published or not. The documents may come from teaching and research institutions in France or abroad, or from public or private research centers.
L'archive ouverte pluridisciplinaire HAL, est destinée au dépôt et à la diffusion de documents scientifiques de niveau recherche, publiés ou non, émanant des établissements d'enseignement et de recherche français ou étrangers, des laboratoires publics ou privés. 


\title{
Self-induced transparency scenario revisited via beat-wave heating induced by Doppler shift in overdense plasma layer
}

\author{
A. Ghizzo, D. DelSarto, T. Réveillé, N. Besse, and R. Klein
}

Citation: Physics of Plasmas 14, 062702 (2007); doi: 10.1063/1.2735123

View online: https://doi.org/10.1063/1.2735123

View Table of Contents: http://aip.scitation.org/toc/php/14/6

Published by the American Institute of Physics

\section{Articles you may be interested in}

On the multistream approach of relativistic Weibel instability. III. Comparison with full-kinetic Vlasov simulations Physics of Plasmas 20, 082111 (2013); 10.1063/1.4817752

Saturation process induced by vortex-merging in numerical Vlasov-Maxwell experiments of stimulated Raman backscattering

Physics of Plasmas 14, 072704 (2007); 10.1063/1.2749715

On the multistream approach of relativistic Weibel instability. I. Linear analysis and specific illustrations Physics of Plasmas 20, 082109 (2013); 10.1063/1.4817750

On the multistream approach of relativistic Weibel instability. II. Bernstein-Greene-Kruskal-type waves in magnetic trapping

Physics of Plasmas 20, 082110 (2013); 10.1063/1.4817751

Multidimensional electron beam-plasma instabilities in the relativistic regime

Physics of Plasmas 17, 120501 (2010); 10.1063/1.3514586

Relativistic laser piston model: Ponderomotive ion acceleration in dense plasmas using ultraintense laser pulses

Physics of Plasmas 16, 083103 (2009); 10.1063/1.3196845

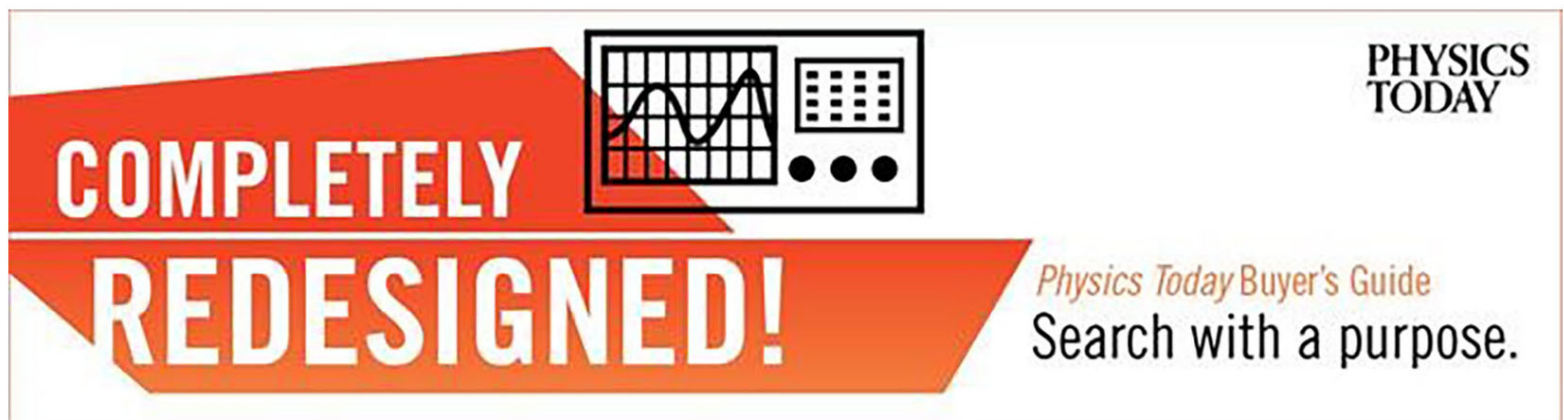




\title{
Self-induced transparency scenario revisited via beat-wave heating induced by Doppler shift in overdense plasma layer
}

\author{
A. Ghizzo, D. DelSarto, T. Réveillé, N. Besse, and R. Klein \\ L.P.M.I.A., Université Henri Poincaré, BP 239, 54506 Vandoeuvre les Nancy Cedex, France
}

(Received 20 December 2006; accepted 6 April 2007; published online 12 June 2007)

\begin{abstract}
Maxwell-fluid simulations on a flat-topped moderately overdense plasma slab (typically $n_{0} / n_{c}$ =1-2) by Berezhiani et al. [Phys. Plasmas 66, 062308 (2005)] \{see also the previous work of Tushentsov et al. [Phys. Rev. Lett. 87, 275002 (2001)]\} were seen to lead to dynamic penetration of an ultrahigh intensity laser pulse into an overdense plasma. Two qualitatively different scenarios for the penetration of laser pulse into the overdense plasma were presented depending on the background density. In the first one, the penetration of laser energy occurs by soliton-like structures moving into the plasma. In the last one, electron cavitation occurs and the penetration is possible over a finite length only. A kinetic extension is made in this paper using Vlasov-Maxwell simulations. Vlasov simulations revealed a rich variety of new phenomena associated with the trapped particle dynamics, which cannot be described in fluid models. Most notably is the observation, during the penetration phase of the pump electromagnetic wave, of a beat-wave heating scenario induced by the Doppler shift on the reflected wave at the (moving) wave front. This beat-wave generates low-frequency acoustic-like electron modes characterized by coherent trapping-type structures in phase space leading to an efficient (nonstochastic) heating process.
\end{abstract}

(C) 2007 American Institute of Physics. [DOI: 10.1063/1.2735123]

\section{INTRODUCTION}

In the high laser intensity regime, the quiver velocity of electrons become relativistic and increasing intensities lead to the relativistic increase of the inertial electron mass and the associated decrease of the effective plasma frequency that may modify the optical properties of the plasma. Relativistic effects allow the wave propagation in homogeneous plasmas with density up to $n_{0}=\gamma_{0} n_{c}$ (where the Lorentz factor of an oscillating electron in the transverse direction is given by $\gamma_{0}=\sqrt{1+a_{0}^{2}}$ and $2 a_{0}^{2}=I \lambda_{0 V}^{2} / 1.368 \times 10^{18}$ for a circularly polarized electromagnetic wave, $I$ being the intensity in $\mathrm{W} \mathrm{cm}{ }^{-2}$ ). This leads to the self-induced transparency scenario (SIT), i.e., the laser propagation through an overdense plasma in which the propagation is classically forbidden (for plasmas having density above the critical density $n_{c}=1.1$ $\times 10^{21} \lambda_{0 V}^{2} \mathrm{~cm}^{-3}$, where $\lambda_{0 V}$ is the laser wavelength in microns).

These relativistic effects and the strongly nonlinear character of the interaction play a fundamental role in determining the dynamical evolution of the plasma. These effects become crucial in technical applications as in the ignition fusion concept proposed by Tabak in Ref. 1.

Recently applying the one-dimensional (1D) Maxwellfluid model, Tushentsov et al. in Ref. 2 charted out two different scenarios for SIT, for moderately relativistic pulses, depending on the background supercritical density. For plasma density $n_{0}$ less than $1.5 n_{c}$, the penetration of the laser pulse occurs in the form of long lived soliton-like structures, while at higher background density $n_{0}>1.5 n_{c}$, the authors have observed the generation of plasma field structures consisting of alternating electron and vacuum regions extended into the plasma over a finite length. These two scenarios were recently confirmed in Ref. 3 by Berezhiani et al., using global Maxwell-fluid simulations. Only circularly polarized laser pulses were considered to simplify the presentation of the physical processes. These simulations have shown that the relativistic laser pulse can considerably reduce the electron density in the region in front of the moving wave front until electron cavitation takes place. This electron cavitation acts as a "wall" making the plasma opaque to the propagation of the electromagnetic pulse. The scenario presented by these authors is however based on a fluid (and cold) approach which do not take into account kinetic effects and in particular the nonlinear electron dynamics.

On the other hand, first kinetic particle-in-cell (PIC) simulations used in Refs. 4 and 5 in this regime of SIT have revealed anomalous longitudinal electron heating, which can crucially change the optical properties of plasma under the action of the laser field. Kinetic effects may then be important and are responsible of the significant absorption of the laser pulse energy. This effect is then outside the domain of the Maxwell-fluid model. Different physical processes were predicted with the help of analytical models and PIC simulations. Vshiukov et al. (see Ref. 6) have characterized the basic features of the interaction of short laser pulse with a thin foil showing harmonic generation, polarization changes and creation of quasistatic magnetic field. A clear indication of the importance of electron kinetic effects in the study of ion acceleration to high energies was also presented in Refs. 7 and 8 using PIC simulations. Thus it seems important to take into account kinetic effects in the study of SIT.

It is generally recognized that PIC codes present a high level of numerical noise and can overestimate the heating of plasma due to their "numerical heating." On the other hand, semi-Lagrangian Vlasov codes display an extremely low 
level of numerical noise and prove to be able to describe fine details of accelerated and trapped particles in phase space met in parametric instabilities such as stimulated Raman scattering or beat-wave acceleration (see Refs. 9). The idea to use Vlasov models to describe SIT at moderate intensities is then natural.

To study SIT we used a semi-Lagrangian VlasovMaxwell code with only one spatial dimension $x \omega_{p e} / c$ in the axial wave vector direction, the relevant phase space being $\left(x \omega_{p e} / c, p_{x} / m_{e} c\right) ; \omega_{p e}$ is the electron plasma frequency and $m_{e}$ is the electron rest mass. The perpendicular motion in the transverse direction is being followed via conservation of the canonical momentum; the plasma is in effect, cold in the transverse direction as far as the Lorentz factor $\gamma$ is concerned. The physical model used here has been presented in Ref. 10 and the numerical scheme in Ref. 11.

We address in this paper the physical mechanism which is responsible for electron heating in the regime of SIT for a moderately overdense plasma layer. An electromagnetic beat-wave is observed in Vlasov simulations which consists of the beating of the incoming laser pulse with its Dopplershifted reflected electromagnetic wave. This process leads to the formation of low-frequency electron acoustic-like waves (EAWs) which, in the nonlinear regime are characterized by trapping structures in the bulk of the distribution function. The concept of this electron heating is a novel version of the beat-wave heating first introduced by Cohen et al. ${ }^{12}$ which may be applied in the case of an overdense plasma. Here the process is produced in a self-consistent way by the Doppler shift introduced by the (moving) wave front.

The outline of the paper is as follows: In Sec. II we present this novel scenario responsible for nonstochastic electron heating in the sense that coherent trapping structures are created in phase space by the beat-wave. Then we present in Sec. III the first numerical results with physical parameters used by Berezhiani et al. presented in Ref. 3 in order to make a comparison with fluid simulations. The case of an initially hot plasma is then investigated in Sec. IV. In Sec. V we have determined the limit value of the electron slab density which makes the plasma opaque to the penetration of the pulse. We let vary the initial plasma temperature and the quiver momentum to study in detail the process in Sec. VI. Section VII concerns the competition process of beat wave heating with electron cavitation including the possibility of ion acoustic waves (IAWs) excitation. Finally we conclude in Sec. VIII.

\section{DOPPLER-SHIFT-INDUCED BEAT-WAVE HEATING}

From our Vlasov simulation data, the penetration by self-induced transparency of an electromagnetic pump wave is characterized by a beat-wave mechanism between the incoming pump wave and its reflected electromagnetic wave.

This beat-wave is attributed to the formation of a discontinuity in the plasma density associated with the penetration of the light into the overdense plasma layer by relativistic effects. During the penetration of the laser pulse into the plasma, a relativistic Doppler shift takes place at the wave front, which acts as a moving mirror. Here it is the beating between the incoming pump with its reflected wave that determines the nature of the low-frequency nonlinear EAWs in terms of wave numbers and frequencies.

Let us denote $v_{F}$ as the propagation velocity. A resonant beat-wave mechanism between the incoming (referred to here as the pump) electromagnetic wave $\left(\omega_{0}, k_{0}\right)$ and the reflected wave $\left(\omega_{r},-k_{r}\right)$ takes place in a region located in front of the wave front and gives rise to a low-frequency nonlinear EAW $\left(\omega_{e}, k_{e}\right)$. Here $k_{r, 0, e}$ is the modulus of the vector $\mathbf{k}_{r, 0, e}$.

We refer to this unusual beat-wave process involving particle trapping in the bulk of the distribution function as Doppler beat-wave heating (DBWH), to emphasize both requirements, the Doppler-shift induced by relativistic effects, and the heating process resulting from this beat wave. This Raman-type process is different from the usual beat wave accelerator concept (BWA) of Tajima and Dawson proposed in Ref. 13 met in laser plasma accelerators, in which a plasma wave $\left(\omega_{\text {pe }}, \mathbf{k}_{0}-\mathbf{k}_{1}\right)$ is created through the beating of two electromagnetic waves $\left(\omega_{0}, \mathbf{k}_{0}\right)$ and $\left(\omega_{1}, \mathbf{k}_{1}\right)$ provided that the separation of the two electromagnetic pulses is equal to the plasma frequency $\omega_{0}-\omega_{1}=\omega_{p e}$ and $\mathbf{k}_{0}-\mathbf{k}_{1}=\mathbf{k}_{p}$, where $k_{p}$ is the wave number of the plasma wave. While BWA calls for a highly underdense plasma $\omega_{p e} / \omega_{0} \ll 1$, which guarantees that the electron plasma wave remains amplified at high phase velocity $v_{\varphi}=\omega_{p e} / k_{p} \simeq c$, DBWH gives rise to a lowfrequency electron trapping mode of weak phase space velocity. This makes particle trapping very efficient since the bulk of the distribution is concerned. The interaction drives initially an electron acoustic wave but nonlinear effects strongly modify the nature of the EAW since the plasma is characterized by trapping structures (here "bumps" rather than "holes") in phase space.

Matching conditions required for the resonance are then given by

$$
\omega_{0}=\omega_{r}+\omega_{e} \text { and } k_{0}=-k_{r}+k_{e} .
$$

The electromagnetic waves (pump plus reflected wave) satisfy the standard linear dispersion relation, for circularly polarized waves,

$$
\omega_{0, r}^{2}=\omega_{p e}^{2}\left\langle\gamma^{-1}\right\rangle+k_{0, r}^{2} c^{2}
$$

in which the mean inverse Lorentz factor is given by

$$
\left\langle\gamma^{-1}\right\rangle=\frac{1}{n_{e}} \int_{L_{1}}^{L_{2}} d x \int_{-\infty}^{+\infty} d p_{e} f_{e} / \gamma_{e} .
$$

For the electron population the Lorentz factor is indeed given by

$$
\gamma_{e}^{2}=1+\frac{p_{x, e}^{2}}{m_{e}^{2} c^{2}}+a^{2}(x, t),
$$

where $a^{2}=e^{2} A_{\perp}^{2} / m_{e}^{2} c^{2}$ and $p_{x, e}$ is the electron momentum ( $e$ and $m_{e}$ are the electron charge and rest mass and $A_{\perp}$ is the transverse vector potential).

In Eq. (3) the integral was computed over the plasma length $L_{2}-L_{1}$ located in the region where electron modes are present. These low-frequency EAWs, induced by the beatwave are driven in a nonlinear way and are characterized by 
the formation of trapping structures (here "bumps") of high density in phase space. The electron mode obeys the following condition:

$$
\omega_{e}=k_{e} v_{F}=\left(k_{0}+k_{r}\right) v_{F} \propto k_{e} v_{\text {th }},
$$

which can be easily obtained by considering a Lorentz transform in frequency, with $v_{\text {th }}$ as the electron thermal velocity. In the frame of the wave front, the frequency of this electron wave is $\omega_{e}^{\prime}=\gamma_{F}\left(\omega_{e}-k_{e} v_{F}\right)=0$, since in this frame both pump and reflected light wave have the same frequency. Here we have $\gamma_{F}=\left(1-\beta_{F}^{2}\right)^{-1 / 2}$ and $\beta_{F}=v_{F} / c$. It must be pointed out that the wave front velocity $v_{F}$ depends on the initial pump intensity, the electron plasma temperature, and the ratio of the electron slab density to the critical density.

In this new regime, the expansion of ions into vacuum is too slow for the electron motion associated with both phases of the laser-plasma interaction to destabilize the electron modes. Because Vlasov simulations show that, with a plasma slab and a fairly strong laser field, the beat-wave process induced by the Doppler shift may grow much faster than the typical time scale of ion motion, our neglect of the ion motion is justified here for the time of simulation. This process is easily observed for short systems and fast electron time scales. The underlying physics of the observed anomalous heating is essentially the generation of these low frequency EAWs in a nonlinear regime which are not Landau damped (and in spite of the high value of the $k_{e} \lambda_{\text {De }}$ parameter). However the frequency range in which the beat mode can be excited is not limited to (nonlinear) EAWs. Ion acoustic waves (IAWs) may also be excited. This excitation however arises when the corresponding ion acoustic velocity $c_{s}$ is very close to the wave front velocity $v_{F}$. The main difficulty in exciting IAW is that the wave front velocity is usually too high to make the coupling with IAWs not possible. The situation is somewhat more favorable (i) when electron cavitation takes place since in that case the wave front velocity is significantly reduced by the cavitation and (ii) in an initially hot plasma allowing higher values of $c_{s}$ which are close to the wave front velocity.

\section{BEREZHIANI'S SIMULATION RESULTS}

Direct comparison with the work of Berezhiani et al. (presented in Ref. 3) is difficult since the fluid model appears as a limit of the Vlasov model when the initial Maxwellian distribution function tends to a Dirac distribution in velocity space. Of course the case of an exact Dirac distribution cannot be used in Vlasov simulations due to a computer burdensome cost required to sample the Dirac-type electron distribution function in phase space. To maintain the connection with the work of Berezhiani et al., ${ }^{3}$ we begin with a similar simulation, a plasma slab of $n_{0} / n_{c}=1.6$ and $a_{0}=\sqrt{2}$ (i.e., for $\left.n_{0} / \gamma_{0} n_{c} \simeq 0.924\right)$ and to an irradiation of $I \lambda_{0 V}^{2}=5.47$ $\times 10^{18} \mathrm{~W} \mathrm{~cm}^{-2} \mu \mathrm{m}^{2}\left(\lambda_{0 V}\right.$ being the pump wavelength in vacuum). This simulation corresponds to Fig. 10 in Ref. 3. Note that the laser intensity is slightly smaller than that used in Berezhiani's work.

We consider an initial plasma temperature of $T_{e}$ $=10 \mathrm{keV}$ (similar results were obtained for a lower plasma

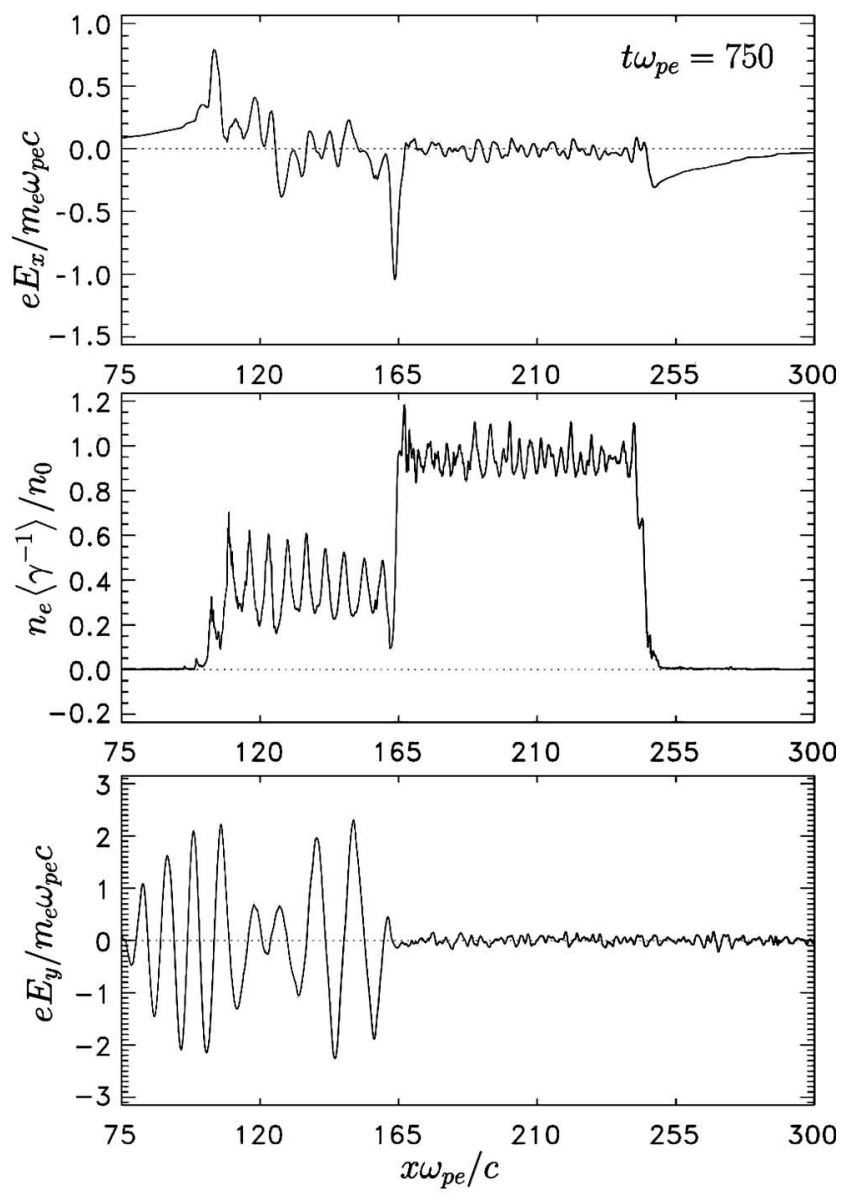

FIG. 1. The figure gives an example of the constitution of the wave front observed in the Vlasov simulation: longitudinal electric field $e E_{x} / m_{e} \omega_{p e} c$ (top), the relative electron density $n_{e}\left\langle\gamma^{-1}\right\rangle / n_{0}$ (middle), and the transverse electric field $e E_{y} / m_{e} \omega_{p e} c$ (bottom) as a function of space $x \omega_{p e} / c$ at the same time $t \omega_{p e}=750$. At the wave front, a discontinuity in the relative electron density (acting as a mirror and leading to a Doppler shift) is clearly visible. The physical parameters are $n_{0} / n_{c}=1.60$ and $a_{0}=\sqrt{2}$. This simulation corresponds to Fig. 10 in Ref. 3. Here the electron plasma temperature is $T_{e}$ $=10 \mathrm{keV}$. Ions are fixed.

temperature of $T_{e}=3 \mathrm{keV}$ ). The physical system consists of a plasma slab of uniform density $n_{0}$. The length of the plasma slab is $L_{\text {slab }}=150 \mathrm{c} / \omega_{p e}$, and is surrounded by a vacuum region of $75 c / \omega_{p e}$ and we take $L_{\text {jump }}=25 c / \omega_{p e}$, where $L_{\text {jump }}$ is a small region over which the density jumps from zero to $n_{0}$. The circularly polarized electromagnetic wave is described by a plane wave and is introduced at the left boundary and propagates (in the $x$ direction) through a region of vacuum onto a slab of uniform plasma. The laser amplitude rises approximately linearly during a time of $t \omega_{p e}=150$ and is then held constant during all the rest of the simulation. The time step is $\Delta t \omega_{p e}=\Delta x \omega_{p e} / c$ is close to 0.027 (i.e., for a ratio of the space spacing over the Debye length $\Delta x / \lambda_{\mathrm{De}} \simeq 0.20$ ). Here the momentum spacing is $\Delta p_{x, e} / m_{e} c \simeq 0.03$.

Figure 1 gives an example of the constitution of the wave front observed in the Vlasov simulation. In the penetration zone of the incident electromagnetic wave inside the overdense plasma a large longitudinal electric field is generated (on top of Fig. 1) with a peak located at the discontinuity of the (relative) electron density $n_{e}\left\langle\gamma^{-1}\right\rangle / n_{0}$ (see the 

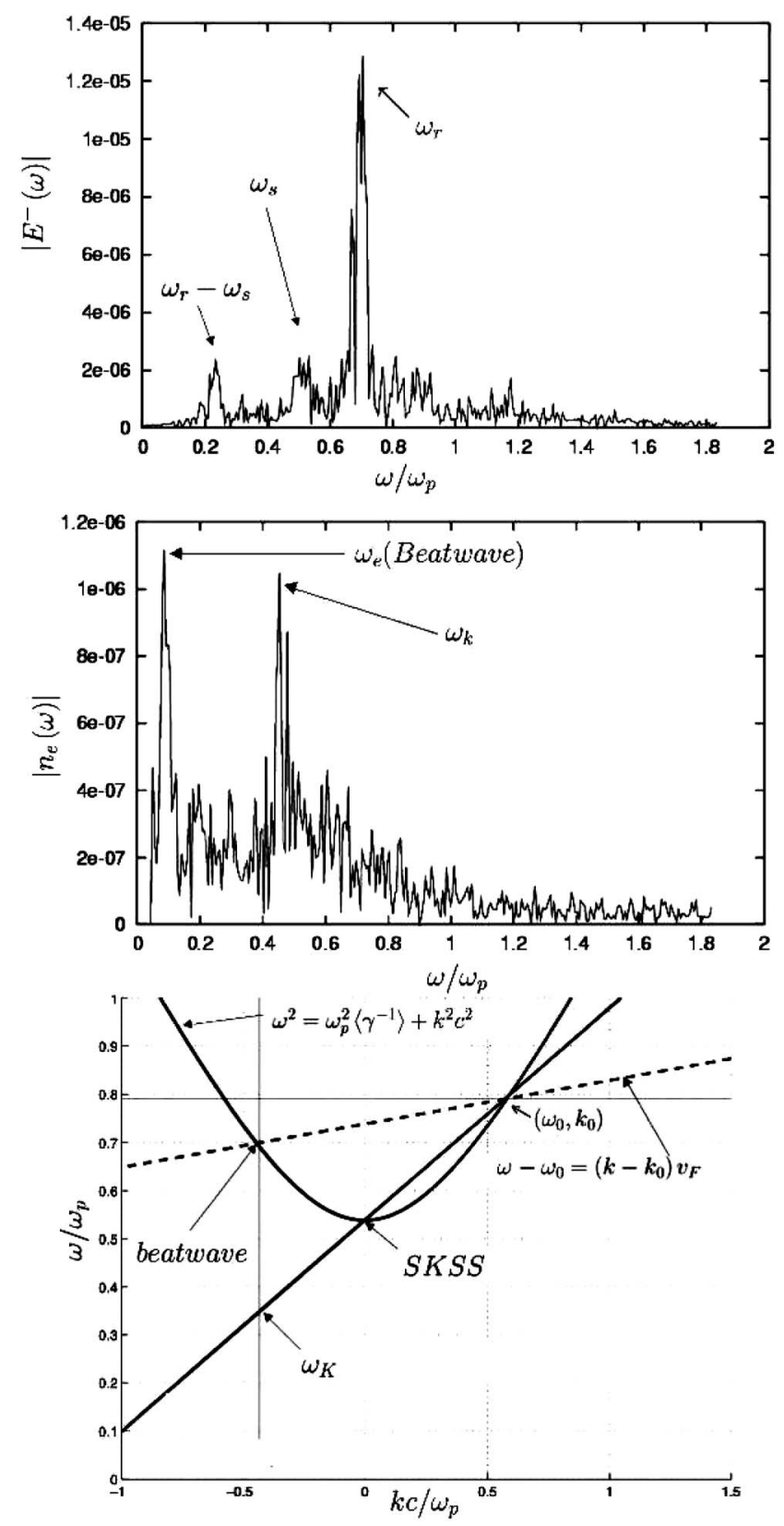

FIG. 2. Display of the backward electromagnetic spectrum in frequency (top panel) and of the frequency spectrum of the electron density (middle panel). The electrostatic spectrum clearly exhibits the beat wave frequency close to $\omega_{e} \simeq 0.09 \omega_{p e}$, but also another mode at $\omega_{K} \simeq 0.45 \omega_{p e}$ related to a KEEN wave. Bottom panel: frequency $\omega / \omega_{p e}$ versus wave number $k c / \omega_{p e}$ diagram illustrating the DBWH mechanism (dashed line) and the excitation of a KEEN wave on the wave vector $k_{e}$ (solid line). The physical parameters are those used in Fig. 1 and correspond to Berezhiani's physical parameters $n_{0} / n_{c}=1.60$ and $a_{0}=\sqrt{2}$.

middle panel). The last plot displays the corresponding transverse electric field $e E_{y} / m_{e} \omega_{p e} c$. We clearly see the formation of the discontinuity in the relative electron density in the middle panel. The measure of this displacement in $x$ at different times gives an estimation of the wave front velocity $v_{F}$.

Figure 2 displays the corresponding spectra in frequency. The top panel corresponds to the reflected electromagnetic spectrum in frequency. Here the frequency resolution is given by $\Delta \omega=0.0040 \omega_{p e}$. The major component of interest in the backward spectrum is the reflected wave located at the frequency $\omega_{r} \simeq 0.700 \omega_{p e}$. Note that in this simulation the pump wave frequency is $\omega_{0}=\left(n_{0} / n_{c}\right)^{-1 / 2} \omega_{p e} \simeq 0.79057 \omega_{p e}$. Taking $\left\langle\gamma^{-1}\right\rangle \simeq 0.30$ (measured in the Vlasov simulation through the data of $f$ ) and $k_{0} c / \omega_{p e} \simeq 0.570$ (which was measured in the $k$ spectrum of the fields), the dispersion relation (2) leads to a value of $\omega_{0} \simeq 0.79050 \omega_{p e}$. The analysis of the $k$ spectrum of the electric field yields to $k_{r} c / \omega_{p e} \simeq 0.435$ for the reflected light and $k_{e} c / \omega_{p e} \simeq 1.005$ for the electron mode. Note also the presence in the backward spectrum of a lower peak (in intensity) at $\omega_{s} \simeq 0.55 \omega_{p e}$ corresponding to a backscattered light emitted not far from the cutoff (we have for the relativistic plasma frequency $\left.\omega_{p e}\left\langle\gamma^{-1}\right\rangle^{1 / 2} \simeq 0.5477 \omega_{p e}\right)$.

The bottom panel displays the frequency spectrum of the electron density which clearly exhibits the beat wave frequency here close to $\omega_{e} \simeq 0.09 \omega_{p e}$, but also another mode at $\omega_{K} \simeq 0.45 \omega_{p e}$. Note that the obtained value of $\omega_{e}$ is very close to the expected value calculated via the data of $v_{F}$, i.e., $\omega_{e}=k_{e} v_{F}=0.09 \omega_{p e}$ since $k_{e}=1.005 \omega_{p e} / c$ and $v_{F} \simeq 0.09 c$ which can be directly estimated by measuring the velocity of the wave front in the curves of the density at different times during the simulation.

An interesting feature of the interaction observed in the frequency spectrum must be pointed out: the plasma response is not a simple driven mode (EAW) but rather seems to be a more complicated state corresponding to the mixing of the nonlinear EAW (with a frequency peak located at $\omega_{e}$ $=0.09 \omega_{p e}$ ) and of a second mode (characterized by a peak in spectrum at $\omega_{K} \simeq 0.45 \omega_{p e}$ ). This second mode is indeed highly nonlinear and inherently kinetic in nature and might be a kinetic electrostatic electron nonlinear (KEEN) wave. First predicted by Afeyan et al. in Ref. 21 in the low laser intensity regime using electrostatic Vlasov simulations and an external independent ponderomotive driver of finite duration, the KEEN waves were also observed in the relativistic regime in Ref. 15. The simulations performed by Afeyan $e t$ al. (see Ref. 21) have shown that long-lived excitations of a KEEN wave, at frequencies at something like a third of the plasma frequency, can indeed be produced if coherently driven up to self-sustained level. The KEEN waves are a novel version of a Bernstein-Greene-Kruskal (BGK) nonlinear wave (presented in Ref. 16) with electrons trapped in the same wave troughs.

To aid in understanding what has happened, we present in the bottom panel in Fig. 2 a graphic version of the different mechanisms met in a simulation for an open plasma using the same parameters. We have plotted the normalized dispersion relation for the circularly polarized electromagnetic wave in solid line. The dashed line corresponds to the DBWH process (i.e., the EAW dispersion relation). The second straight-line crossing the origin (in solid line) corresponds to the dispersion relation of the KEEN wave (which was determined numerically using a set of periodic VlasovMaxwell simulations using the same external ponderomotive driver and looking at the corresponding plasma response). We see in the bottom panel that both electron beat mode and KEEN wave were thus excited at the same $k_{e}$ wave vector in the causal Vlasov simulation. The frequencies correspond, 
respectively, to the numerical values $\omega_{e}=0.09 \omega_{p e}$ and $\omega_{K}$ $=0.45 \omega_{p e}$ observed in the electrostatic spectrum.

Note also that around the origin a stimulated KEEN slab scattering (SKSS), first introduced in Ref. 15, may also be excited in the case of SIT. This process may be observed in the asymptotic regime (i.e., when the laser pulse has crossed all the plasma layer). This process is characterized by the fact that the backscattered wave was always found to be driven near critical, i.e., at the frequency close to the value $\omega_{s}=\omega_{p e}\left\langle\gamma^{-1}\right\rangle^{1 / 2}$ (here the corresponding value is close to $\omega_{s}$ $\simeq 0.54 \omega_{p e}$ and this value is clearly visible in the top panel in Fig. 2 in the backscattered spectrum). This feature of the instability was first seen in a simulation using the PIC code by Nikolic et al. in Ref. 17 using PIC simulations and reexamined by Ghizzo et al. in Ref. 15. This feature is linked to the existence of a radiating plasma cavity which determines the resonance conditions by imposing discrete values (multiples of the half-wavelength) for the wave number of the confined electromagnetic daughter wave.

We focus now on the features of the reflected electromagnetic wave. We can also obtain a good estimation of the reflected frequency due to the Doppler shift produced by the discontinuity in the relative density. An analytical expression of the reflected physical quantities (wave vector and frequency) due to the relativistic Doppler shift was already obtained in Ref. 5, we have here

$$
\begin{aligned}
& \frac{\omega_{r}}{\omega_{0}}=\frac{1+\beta_{F}^{2}-2 \beta_{F} / \beta_{0}}{1-\beta_{F}^{2}}, \\
& \frac{k_{r}}{k_{0}}=\frac{1+\beta_{F}^{2}-2 \beta_{F} \beta_{0}}{1-\beta_{F}^{2}},
\end{aligned}
$$

where $\beta_{F}=v_{F} / c$ and $\beta_{0}=\omega_{0} / k_{0} c$. Using $\beta_{0}$ $=\left(\omega_{0} / \omega_{p e}\right) /\left(k_{0} c / \omega_{p e}\right)=1.385$ and $\beta_{F}=0.09$, Eq. (5) gives the value $\omega_{r}=0.690 \omega_{p e}$ in good agreement with the numerical value of $\omega_{r}$ observed in the Vlasov simulation. From Eq. (6) the corresponding wave vector is then $k_{r}=0.420 \omega_{p e} / c$, close to the numerical value of $k_{r}=0.435 \omega_{p e} / c$.

By storing the electromagnetic fields at a position in the vacuum region surrounding the plasma at each time step, the reflection and transmission rates can be evaluated by $R$ $=\Phi_{r} / \Phi_{i}$ and $T=\Phi_{t} / \Phi_{i}$ where $\Phi_{i}$ is the incident Poynting vector flux, $\Phi_{r}$ is the reflected one, and $\Phi_{t}$ is the transmitted one. During the penetration phase, the measured reflection rate in simulation was found to be $R_{\text {num }} \simeq 67 \%$ in good agreement with the theoretical value of $R=k_{r} \omega_{r} / k_{0} \omega_{0}$ $\simeq 67.7 \%$.

We have also estimated the laser energy absorption in the second phase of the instability when the laser pulse has crossed all the plasma layers. By monitoring the reflected laser flux at the left boundary and the transmitted laser flux at the right hand boundary in vacuum, we have obtained an estimation of $R_{\text {num }} \simeq 5 \%$ and $T_{\text {num }} \simeq 72 \%$, i.e., an estimation of the absorption of $A_{\text {num }}=23 \%$ in the second phase of the instability. These results show that the plasma slab becomes transparent (the corresponding reflection coefficient rate $R$ decreasing strongly) to the laser pulse after the wave front has crossed all the plasma layer. In this asymptotic regime, SKSS is then the dominant process.

While in Berezhiani's work the reflected part of the radiation carries up to $90 \%$ of the incident energy, the Vlasov model indicates a weaker value in the penetration phase due to stronger absorption. For this chosen value of the plasma slab width, electron cavitation is not observed in simulation for the plasma slab width we have chosen here. Vlasov simulations show strong electron heating even at weaker temperature which seems to indicate the importance of the DBWH process.

\section{DBWH FOR A HOT PLASMA}

The basic concept of this particle heating process is that, for a moderately overdense plasma layer, there is a beating between the electromagnetic pump with its Dopplerdownshifted reflected wave in a region located in front of the moving wave front. In this region the laser pulse meets an underdense plasma (due to relativistic effects) in which Raman-type three-wave parametric instabilities are now possible involving EAWs.

DBWH differs however radically from the conventional hole boring scenario or forward motion of the critical surface caused by the action of the ponderomotive pressure where the wave propagation is only possible by the pump light pressure. This last mechanism introduced Denavit et al. in Ref. 4 involves the ponderomotive force associated with the high intensity laser light. Indeed the laser pulse acting as a piston drives a collisionless ion shock wave into the plasma slab which reflects the ions. The conventional Denavit's scenario seems to be inappropriate in the case of plasmas of moderate density. Here a strong heating mechanism is generated which modifies the optical properties of the plasma and in particular propagation of the laser pulse inside the plasma. It must be pointed out, that to be an efficient heating process, the plasma mode must not to be suppressed by the high Landau damping of any SRS-like processes in high plasma temperature. It is well known that the growth-rates of SRS-type instabilities are significantly reduced for high temperature. However the plasma response seems in that case to be appropriate since these low-frequency electron modes (nonlinear EAWs) are weakly affected by Landau's damping since they are driven in a nonlinear regime.

We choose to increase the electron temperature to be a value of $T_{e}=400 \mathrm{keV}$ in order to study the behavior of the electron response at high temperature (we have taken for the density of the plasma slab a value of $\left.n_{0} / n_{c}=2\right)$. For allowing the penetration of the electromagnetic pump wave, we have also increased the quiver momentum of the pump wave to the value $a_{0}=\sqrt{5}$, i.e., corresponding to an intensity of $I$ $=1.368 \times 10^{18}\left(a_{0} \sqrt{2}\right)^{2} \simeq 1.37 \times 10^{19} \mathrm{~W} \mathrm{~cm}^{-2}$ for a $1 \mu \mathrm{m}$ wavelength.

Our observations differ from previous results obtained by Maxwell-fluid simulations in Ref. 3 which seem to indicate a limit value of the background density close to $n_{0}$ $=1.5 n_{c}$, leading to a different regime of the penetration of the laser pulse. 

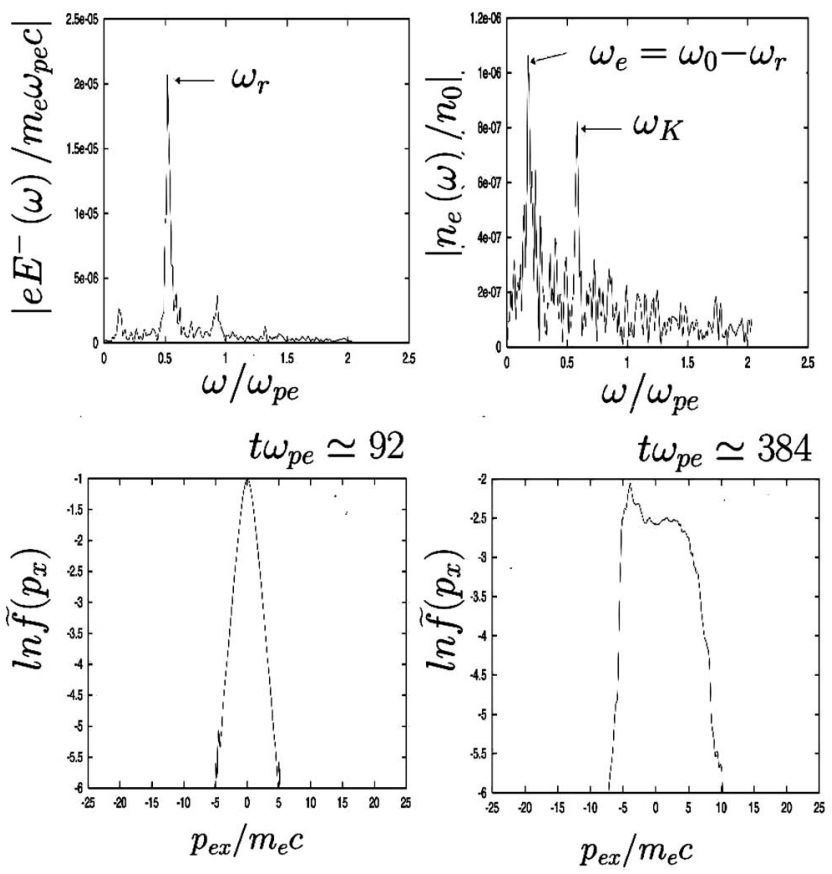

FIG. 3. For a hot plasma of electron temperature of $T_{e}=400 \mathrm{keV}$ and fixed ions. Top: electromagnetic and electrostatic spectra in frequency for physical parameters $n_{0}=2 n_{c}$ and $a_{0}=\sqrt{5}$. The reflected Doppler-shifted electromagnetic wave (top left panel), and the low-frequency acoustic-like electron wave (top right panel) are clearly visible. Bottom: mean distribution function versus $p_{e x} / m_{e} c$ at different times during the interaction. The bottom left panel shows the initial plasma state when the laser has not reached the plasma slab while the bottom right corresponds to the phase of the penetration when the acoustic-like electron mode (beat wave mode) is well established in the plasma.

Figure 3 displays the electromagnetic and electrostatic spectra. The upper panels correspond, respectively, to the backward light spectrum and to the spectrum of the electron density calculated in the time domain of $\left[0,600 \omega_{p e}^{-1}\right]$. The top left panel exhibits a dominant peak at $\omega_{r} \simeq 0.53 \omega_{p e}$ in good agreement with the theoretical value given by Eq. (5) of $\omega_{r}$ $=0.526 \omega_{p e}$, showing the importance of the Doppler shift. Using (6) the corresponding value of the wave vector is then $k_{r}=0.511 k_{0} \simeq 0.29 \omega_{p e} / c$. We have here for the pump frequency $\omega_{0} / \omega_{p e}=\sqrt{n_{0} / n_{c}}=0.707$ and $k_{0} c / \omega_{p e}=0.58$. Using $\left\langle\gamma^{-1}\right\rangle \simeq 0.16$, the value obtained from the (linear) dispersion relation is $\omega_{0}=\left(\omega_{p e}^{2}\left\langle\gamma^{-1}\right\rangle+k_{0}^{2} c^{2}\right)^{1 / 2}=0.704 \omega_{p e}$, in good agreement with the numerical value obtained in spectrum. The wave front velocity used in Eqs. (5) and (6) is about $v_{F}$ $\simeq 0.22 c$.

The beat wave process arises when the matching conditions (1) are satisfied. Here we have obtained $\omega_{0}(0.707)$ $=\omega_{r}(0.50)+\omega_{e}(0.207)$ in $\omega_{p e}$ units in frequencies and $k_{0}(0.58)=-k_{r}(-0.30)+k_{e}(0.88)$ in $\omega_{p e} / c$ units in wave vectors.

In the upper right panel in Fig. 3, the electrostatic spectrum illustrates the occurring of the trapping EAW mode in plasma at $\omega_{e}=0.19 \omega_{p e}$ but also another nonlinear contribution at the frequency $\omega_{K}=0.59 \omega_{p e}$, which however remains at a weak level in simulation and may be attributed to a KEEN wave, driven near its self-sustained level.

The bottom panels in Fig. 3 display the mean distribu-
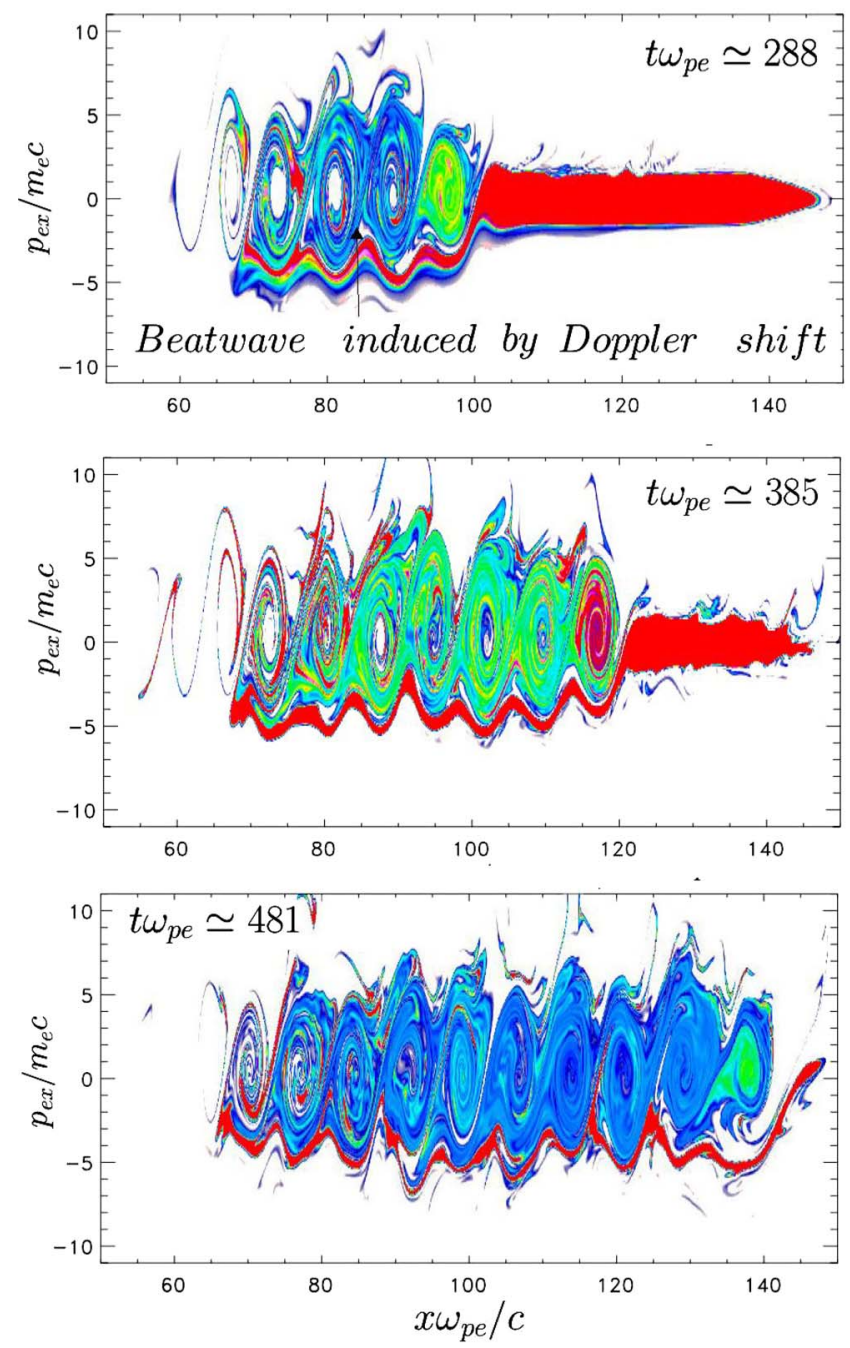

FIG. 4. (Color online) For a uniform and hot plasma with an electron temperature of $T_{e}=400 \mathrm{keV}$ and fixed ions, we show the representation of the electron distribution function in phase space for a numerical simulation carried out with $n_{0}=2 n_{c}$ and $a_{0}=\sqrt{5}$. The panels show the vortices corresponding to trapped acoustic-like electron mode induced by the Doppler shift of the incoming pump wave after reflection.

tion function versus $p_{e x} / m_{e} c$ at different times during the interaction. The function has been averaged over the spatial interval $\left[80 c / \omega_{p e}, 120 c / \omega_{p e}\right]$. The bottom left panel shows the initial plasma state when the laser has not reached the plasma slab. The bottom right panel corresponds now to the phase of the penetration when the acoustic-like electron mode is well established in the plasma. We clearly see the formation of a strong return current and the strong deformation of the distribution function due to DBWH.

Figure 4 gives an example of detailed phase space information available from a Vlasov code such as ours. We focus on the beginning of the evolution showing the state of the plasma characterized by the growth of the DBWH process. The figures show the behavior of the plasma, at three different times during this phase. The beating of this pump with the reflected wave leads to the formation of the EAW mode in the nonlinear regime. As the electromagnetic wave penetrates inside the plasma by SIT, new structures are created and their creation is associated with the propagation velocity 

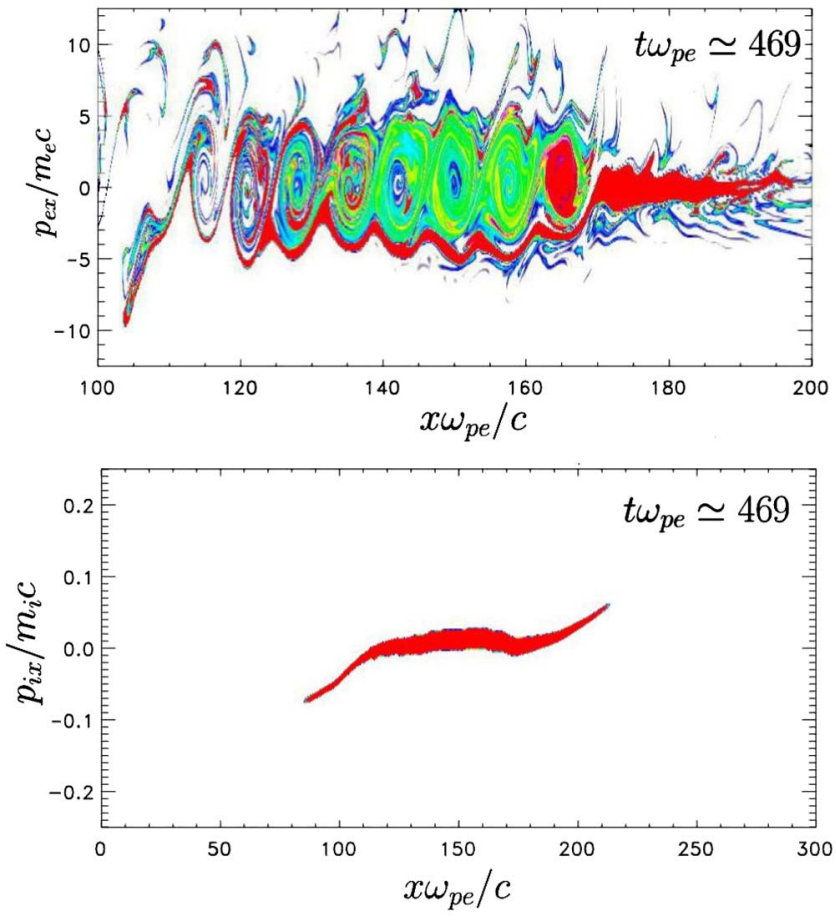

FIG. 5. (Color online) For a uniform and hot plasma with an electron temperature of $T_{e}=400 \mathrm{keV}$ the figure shows, on the top panel, the behavior of the electron distribution function in phase space resulting from a Vlasov simulation carried out with $n_{0}=2 n_{c}$ and $a_{0}=\sqrt{5}$ with mobile ions. Similar behavior was obtained as in Fig. 3 (with fixed ions) in spite of ion motion. The corresponding ion distribution function in phase space is shown in the bottom panel. The bottom panel illustrates the ion dynamics and the ion expansion into vacuum.

of the wave front close to $v_{F} / c=0.22$. For the considered time interval, the KEEN excitation does not arise and we observe just the EAW mode. At $t \omega_{p e} \simeq 481$, the plasma exhibits the formation of ten vortices for a plasma length of $72.5 \mathrm{c} / \omega_{p e}$ or $6.4 \lambda_{0}$ leading to a plasma mode of length of $\lambda_{e} \omega_{p e} / c=7.25$ or to $k_{e} c / \omega_{p e}=0.86$ in good agreement with the expected value.

In this previous example, ion dynamics play a minor role in comparison with the ion collisionless shock predicted in the Denavit's scenario when light pressure is the dominant effect.

Let us now discuss whether allowing movement of the ions with realistic charge to mass ratios will significantly alter DBWH. It is well known that fixed ions act to prevent the electron expansion. The effect of mobile ions is to somewhat modify the plasma edges over several pump wavelengths without significantly changing the structure of the ambipolar field. The test simulation was carried out using an ion-electron mass ratio of $m_{i} / m_{e}=1836$ and an initial ion temperature of $T_{i}=20 \mathrm{keV}\left(m_{i}\right.$ being the hydrogen mass and we used $Z=1)$. Physical parameters are unchanged, i.e., $n_{0} / n_{c}=2$ and $a_{0}=\sqrt{5}$ (which are identical to those used in Figs. 3 and 4). Figure 5 displays the electron distribution function in the phase space when ion motion is taken into account. Contrary to the previous case, the wave front velocity $v_{F}$ was found to be slightly weaker than the value corresponding to fixed ions where we have obtained a value close to $v_{F} \simeq 0.2 c$. This value is higher than the estimation of the velocity of the pushing front by the simple pressure equilibrium given in Ref. 4 by Denavit et al., which in that case can be expressed easily as

$$
\frac{v_{F}}{c}=\left(\frac{n_{c}}{n_{0}} \frac{m_{e}}{m_{i}} a_{0}^{2}\right)^{1 / 2}
$$

which leads here to a value of $v_{F}=0.036 c$. In the early stage of the penetration dynamics, vortices induced by the beat wave were observed in phase space.

The bottom panel in Fig. 5 illustrates the ion dynamics and the ion expansion into vacuum. As might be expected, the addition of realistic ions has little effect on the DBWH process if the laser flux is high enough.

\section{DBWH AND ELECTRON DENSITY LIMIT VALUE}

We focus now on the production of the beat wave for a range of plasma densities and in particular on the limit value of the density for which the plasma becomes opaque. A series of numerical simulations was carried out in the case of a homogeneous and hot plasma slab to confirm the existence of DBWH and the generation of nonlinear EAWs for a wide range of values of $n_{0} / n_{c}$. We have analyzed in detail the existence of a limit value in density when the laser flux and plasma temperature are fixed. We choose to keep constant the parameter $\left\langle\gamma^{-1}\right\rangle_{0} \simeq 0.368$ [the initial value of the $\left\langle\gamma^{-1}\right\rangle$ quantity], which corresponds to $a_{0}=1.75$ and to an initial plasma temperature of $T_{e}=400 \mathrm{keV}$. We have also reduced the plasma slab length to a somewhat smaller value namely, $L_{\text {slab }}=60 c / \omega_{p e}$ to reduce the effect of electron cavitation (see Sec. VII), which may prevent the penetration of the pump wave. We will deal with densities both less and greater than 1.5 times the critical density. For the simulations we have considered here, the physical system consists of a plasma layer of uniform density of length $L_{\mathrm{slab}}$, surrounded by a vacuum region of length $L_{\mathrm{vac}}=60 \mathrm{c} / \omega_{p e}$ on both sides. The total length of the system is then $L=L_{\text {slab }}+2 L_{\text {vac }}+2 L_{\text {jump }}$ $=210 \mathrm{c} / \omega_{p e}$ using a jump in density of $L_{\text {jump }}=15 \mathrm{c} / \omega_{p e}$. We have injected a circularly polarized plane wave on the left side of the simulation box. In these simulations, $n_{0} / n_{c}$ was varied from 1.0 to 3 .

As can be seen in Fig. 6, which displays the normalized wave front velocity $v_{F} / c$ as a function of the ratio $n_{0} / n_{c}$, it is possible to excite DBWH over a wide range of densities until a limit value. Thus for a given value of the parameter $\left\langle\gamma^{-1}\right\rangle$, the penetration of the incident electromagnetic pulse is only possible well below a threshold in density. The solid line was obtained by estimating directly the wave front velocity as a function linearly decreasing in $n_{0} / n_{c}$, i.e., $v_{F} / c=\left\langle\gamma^{-1}\right\rangle$ $\times\left(\left\langle\gamma^{-1}\right\rangle_{0}^{-1}-n_{0} / n_{c}\right)$. The numerical estimation values obtained from the actual Vlasov simulations are represented by crosses on the curve. These values were measured by analyzing the distribution function behavior in phase space. The close agreement between the straight line and the numerical results shows that this wave front velocity decreases in a linear way as the function of the ratio $n_{0} / n_{c}$ until the plasma layer becomes opaque. 


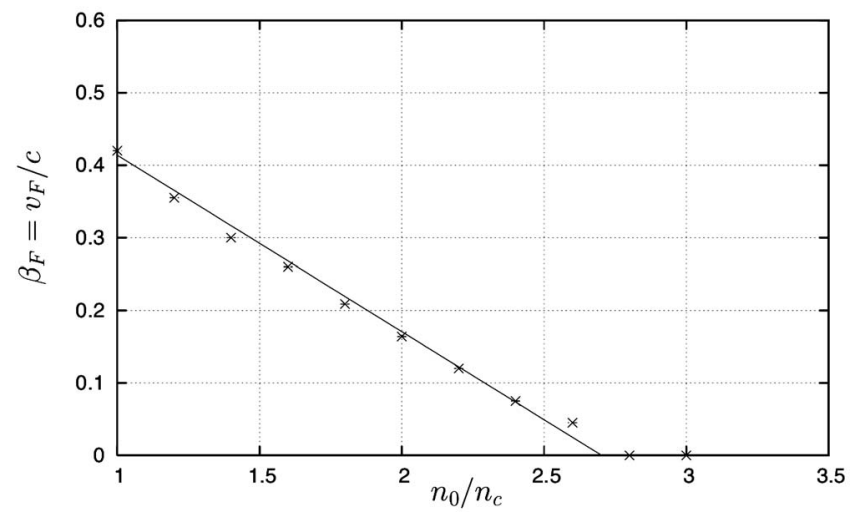

FIG. 6. Normalized wave front velocity $v_{F} / c$ as a function of the ratio $n_{0} / n_{c}$ : it is possible to excite DBWH over a wide range of densities until a limit value. Thus for a given value of the parameter $\left\langle\gamma^{-1}\right\rangle$, the penetration of the incident electromagnetic pulse is only possible well below a threshold in density. The solid line was obtained by estimating directly the wave front velocity as a function linearly decreasing in $n_{0} / n_{c}$, i.e., $v_{F} / c=\left\langle\gamma^{-1}\right\rangle$ $\times\left(\left\langle\gamma^{-1}\right\rangle_{0}^{-1}-n_{0} / n_{c}\right)$. The close agreement between the straight line and the Vlasov numerical results (indicated by crosses) shows that this wave front velocity decreases in a linear way as the function of the ratio $n_{0} / n_{c}$ until the plasma layer becomes opaque to the laser pulse.

To further investigate the possibilities of heating, we now present in detail the result of two simulations in order to make a comparison. We choose to begin with a run which was performed with the physical parameters $n_{0} / n_{c}=1$; ions being fixed. The analysis of the electromagnetic spectrum, shown on the top left panel in Fig. 7, will give confirmation of this beat wave. Indeed the spectrum of the reflected light, measured in the time interval $\left[0,500 \omega_{p e}^{-1}\right]$ (not shown here)
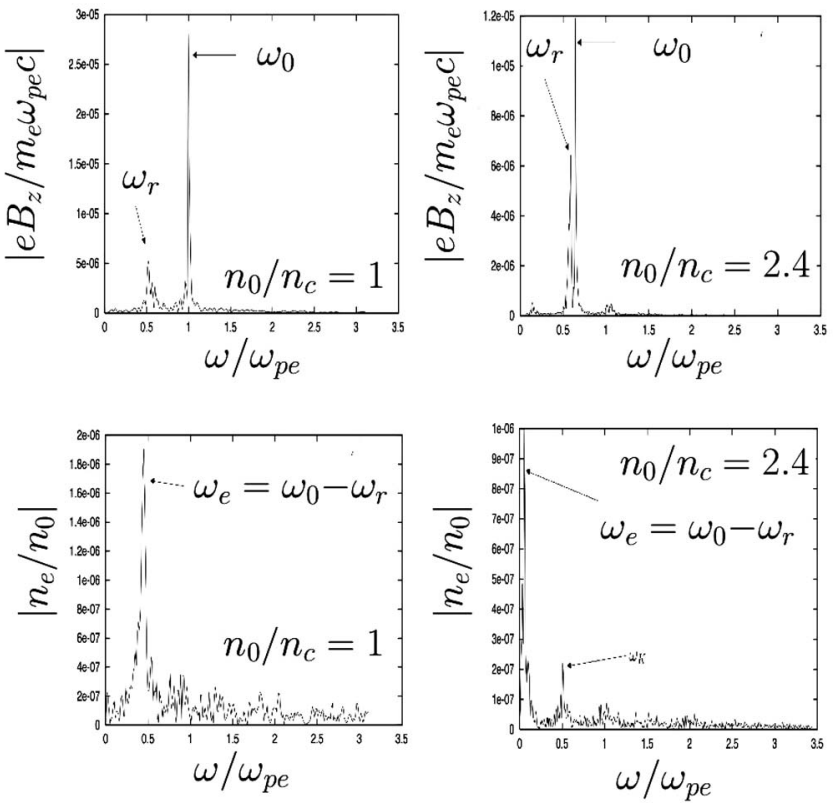

FIG. 7. Electromagnetic and electrostatic spectra in frequency for a numerical simulation of $n_{0}=1 n_{c}$ and fixed ions (left panel) and for $n_{0}=2.4 n_{c}$ (right panels). First during the penetration of the incident wave, we see clearly that the DBWH process takes place and that the beat frequency decreases with $n_{0} / n_{c}$. We choose to keep constant the parameter $\left\langle\gamma^{-1}\right\rangle_{0} \simeq 0.368$ [the initial value of the $\left\langle\gamma^{-1}\right\rangle$ quantity], which correspond to $a_{0}=1.75$ and to an initial plasma temperature of $T_{e}=400 \mathrm{keV}$. exhibits the reflected frequency at $\omega_{r}=0.530 \omega_{p e}$ while the numerical corresponding wave vector is $k_{r} c / \omega_{p e}=0.189$. The theoretical values for the incoming pump wave, given by the dispersion relation (2) are of course $\omega_{0}=1.00 \omega_{p e}$ for a value of the wave number and $k_{0} c / \omega_{p e}=0.868$. An exact estimation of the reflected wave in terms of wave number and frequency is also possible. Using $\beta_{0}=\left(\omega_{0} / \omega_{p e}\right) /\left(k_{0} c / \omega_{p e}\right)=1.152$ and $\beta_{F}=0.42$, Eq. (5) gives the value $\omega_{r}=0.54 \omega_{p e}$, in good agreement with the numerical value of $\omega_{r}$ observed in the Vlasov simulation. From Eq. (6) the corresponding wave vector is then $k_{r}=0.219 \omega_{p e} / c$, close to the numerical value of $k_{r}$.

The EAW mode has then a wave vector close to $k_{e}=k_{0}$ $+k_{r}=1.057 \omega_{p e} / c$ and its frequency $\omega_{e}$ obeys the condition $\omega_{e} / \omega_{p e}=\left(k_{e} c / \omega_{p e}\right)\left(v_{F} / c\right)=1.057 \times 0.42=0.45$ well in agreement with the value obtained by the matching condition in frequency $\omega_{e}=\omega_{0}-\omega_{r} \simeq 0.47 \omega_{p e}$. This value is confirmed in the electrostatic spectrum computed in the time interval $\left[0,500 \omega_{p e}^{-1}\right]$ and shown in the bottom left panel in Fig. 7. The curve exhibits a dominant peak at $\omega_{e}=0.46 \omega_{p e}$.

We now use $n_{0} / n_{c}=2.4$, i.e., a value near the threshold of density where the instability disappears. We expect to obtain a weaker value of the wave front velocity. Other physical parameters were kept fixed. The pump frequency is then $\omega_{0}=0.645 \omega_{p e}$. Using $\left\langle\gamma^{-1}\right\rangle \simeq 0.245$, the value given by the linear dispersion relation of electromagnetic waves in plasma is $\omega_{0}=\left(\omega_{p e}^{2}\left\langle\gamma^{-1}\right\rangle+k_{0}^{2} c^{2}\right)^{1 / 2}=0.644 \omega_{p e}$. Corresponding electromagnetic spectrum (top right panel) as well as the electrostatic spectrum of the electron density (bottom right panel) are presented in Fig. 7 and spectra have now been computed in the time interval $\left[0,850 \omega_{p e}^{-1}\right]$. This electromagnetic spectrum indicates the beating of both electromagnetic waves, in particular the reflected wave can be clearly observed at the frequency $\omega_{r} \simeq 0.58 \omega_{p e}$, while the electron mode is close to $\omega_{e} \simeq 0.06 \omega_{p e}$.

The corresponding three-wave parametric instability arises with the matching conditions (1), i.e., $\omega_{0}=\omega_{r}+\omega_{e}$ (here $0.645=0.587+0.068$ in $\omega_{p e}$ units) and $k_{0}=-k_{r}+k_{e}$ (with $k_{0} c / \omega_{p e}=0.413, k_{r} c / \omega_{p e}=0.315$ and $\left.k_{e} c / \omega_{p e} \simeq 0.728\right)$. From Eqs. (5) and (6) and using $v_{F} / c=0.076$ we obtain $\omega_{r}$ $=0.589 \omega_{p e}$ and $k_{r}=0.319 \omega_{p e} / c$ in good agreement with the numerical values.

Here the objective is to see how the instability is modified when we increase the density of the plasma slab. First in both situations the beat wave can be clearly observed, however we see that the beat frequency, i.e., the frequency of the nonlinear EAW mode decreases strongly when we approach the limit value of the density. Above this limit value $n_{0}$ $\simeq 2.7 n_{c}$ the beating of both electromagnetic waves ceases since both waves now have the same frequency (the wave front velocity being equal to zero).

It is also convenient to analyze the DBWH process in phase space in which the trapped particle dynamics may be observed. Figure 8 shows the behavior of the electron distribution function in phase space. The figure clearly shows the formation and growth of vortices in phase space induced by the beating of both electromagnetic waves. The upper panel corresponds to the case where $n_{0} / n_{c}=1$ while the bottom panel is related to a higher value of 2.40 of the ratio $n_{0} / n_{c}$. While in the upper panel (at time $t \omega_{p e}=269.2$ ), the wave 

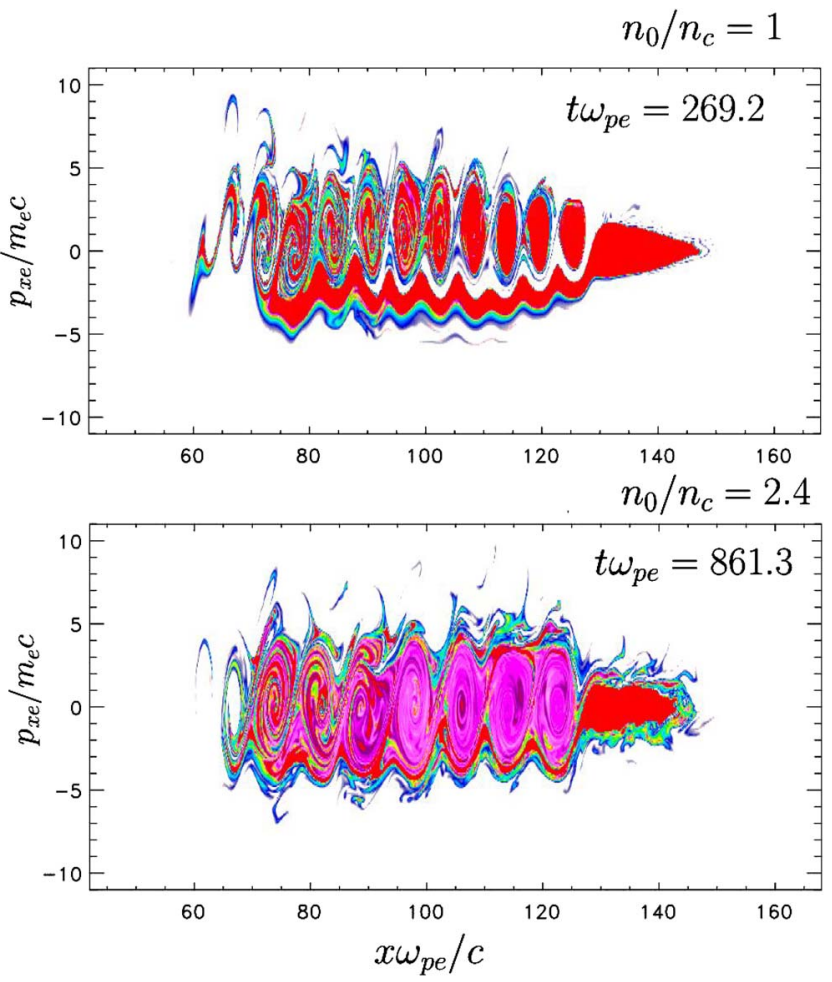

FIG. 8. (Color online) Behavior of the electron distribution function in phase space. The figures clearly show the formation and growth of vortices in phase space induced by the beating of both electromagnetic waves. The upper panel corresponds to the case where $n_{0} / n_{c}=1$ while the bottom panel is related to a higher value of 2.40 of the ratio $n_{0} / n_{c}$. The wave front reaches the same position but at different times.

front has reached the position $x \omega_{p e} / c \simeq 130$, i.e., for a value of the electron density close to the critical density (and with a wave-front velocity close to the maximum value of $v_{F}$ $=0.43 c$ ), these trapping structures decelerate when the density increases. The wave-front velocity settles to a value of $v_{F}=0.075 c$ when $n_{0}=2.4 n_{c}$. As one can better see in the phase space, the wave front is located at the same place (i.e., at $\left.x \omega_{p e} / c \simeq 130\right)$ but now at time $t \omega_{p e}=861.3$, when we choose a high value of the ratio of the electron density to the critical density.

From the distribution function, we have estimated the corresponding wave vector $k_{e}$ of the electron mode close to $k_{e} c / \omega_{p e}=1.08$, for the upper panel, i.e., using $n_{0} / n_{c}=1$. The central region indeed presents ten vortices for a plasma width of $L \omega_{p e} / c=58$, i.e., ten wavelengths, the wavelength of the electron mode is thus close to $\lambda_{e}=5.80 \mathrm{c} / \omega_{p e}$ or equivalently $k_{e}=2 \pi / \lambda_{e}=1.08 \omega_{p e} / c$. This result was in good agreement with the predicted value $k_{e}=1.057 \omega_{p e} / c$ which verifies the matching conditions required for the three-wave parametric instability resonance.

The bottom panel in Fig. 8, corresponding to $n_{0} / n_{c}$ $=2.4$, exhibits seven vortices at time $t \omega_{p e}=861.3$. The corresponding wavelength $\lambda_{e}$ is close to $8 c / \omega_{p e}$ or equivalently $k_{e}=0.785 \omega_{p e} / c$, in that case the resonance arises for $n_{0} / n_{c}$ $=2.4$ when $k_{0}=-k_{r}+k_{e} \quad\left(0.415=-0.315+0.728\right.$ in $\omega_{p e} / c$ units).

Thus it is important to note that our Vlasov simulations show the significant role played by such a DBWH process in the penetration dynamics of the pump pulse inside the plasma layer. This happens regardless of the background density whether it is low $\left(n_{0} \leqslant 1.5 n_{c}\right)$ or high $\left(n_{0} \geqslant 1.5 n_{c}\right)$. It is shown that for an initial value of the parameter $\left\langle\gamma^{-1}\right\rangle_{0}$, the intensive self-induced transparency of the plasma layer can take place only if the plasma density is smaller than a limit value given by the condition $n_{0}\left\langle\gamma^{-1}\right\rangle_{0} / n_{c}=1$ where the wave front velocity $v_{F}$ tends then to zero.

\section{INFLUENCE OF THE TERM $\left\langle\gamma^{-1}\right\rangle$ ON THE DBWH PROCESS}

Previous Vlasov simulations have revealed a strong electron heating mechanism in the case of the penetration of an intense laser pulse into a slightly overdense plasma layer. These simulations have shown that a quasi-instantaneous heating of electrons accompanies the propagation of the wave front as it moves inwards. In particular this heating mechanism clearly was an identification for the limitation of hydrodynamical models in this regime of interaction.

In this section the emphasis is now put on the influence of the $\left\langle\gamma^{-1}\right\rangle$ factor on the velocity of the wave front propagation inside the plasma layer. Most of the results presented here were obtained with fixed ions in order to clarify the electron dynamics. Since kinetic effects or more accurately the plasma temperature as well as the action of the ponderomotive force on the penetration of the laser pulse may modify the $\left\langle\gamma^{-1}\right\rangle$ term, we first study the effect of the plasma temperature $T_{e}$, keeping $n_{0} / n_{c}$ and $a_{0}$ fixed.

The DBWH process depends on the initial plasma temperature. In order to address this point, we perform a set of numerical simulations in which we let the plasma temperature $T_{e}$ vary. We use physical parameter identical to those previously used in Fig. 3 and in Fig. 4, namely $n_{0} / n_{c}=2$ and $a_{0}=\sqrt{5}$. Since the observation of the DBWH mechanism is greatly facilitated in short system, we keep $L_{\text {slab }} \omega_{p e} / c=60$ for the plasma slab width.

We have examined the trapped particle dynamics for two smaller values of the electron plasma temperature namely $T_{e}$ of $10 \mathrm{keV}$ and $200 \mathrm{keV}$. The data of the electromagnetic and electrostatic spectra look generally very similar to that of Fig. 3 (so for brevity we do not present them here), and verify the basic proposition that matching conditions (1) are fulfilled.

Figure 9 show the behavior of the plasma, at three different times. As the electromagnetic wave penetrates inside the overdense plasma by SIT, new vortices are created and the propagation velocity of the wave front is close here to $v_{F}=0.09 c$. At time $t \omega_{p e}=861.3$, we clearly see the formation of ten vortices for the plasma length of $65.3 \mathrm{c} / \omega_{p e}$, which corresponds to $10 \lambda_{e}$. The corresponding wave vector is then $k_{e} c / \omega_{p e}=0.960$ which is close to the expected value. DBWH arises when matching conditions (1) are satisfied (here this corresponds to $\omega_{0}=\omega_{r}+\omega_{e}$ i.e., $0.707=0.621+0.086$ in $\omega_{p e}$ units and $k_{0}=-k_{r}+k_{e}$ or $0.565=-0.453+1.010$ in $\omega_{p e} / c$ units). Note that the corresponding values in frequency and wave vector for the Doppler-shifted electromagnetic wave, using (5) and (6) are then $\omega_{r}=0.615 \omega_{p e}$ and $k_{r} c / \omega_{p e}=0.445$, in good agreement with numerical values. 

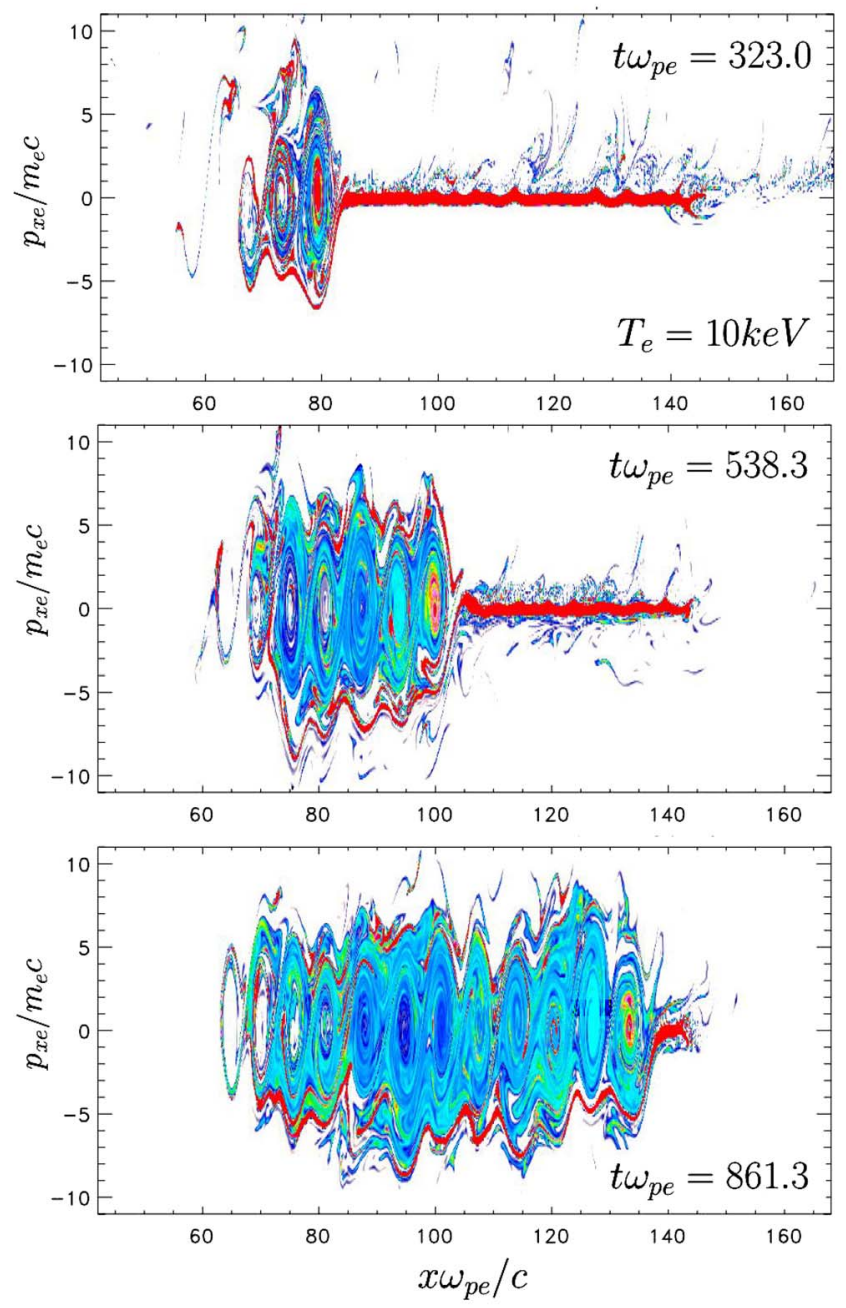

FIG. 9. (Color online) Behavior of the electron distribution function in phase space resulting from a Vlasov simulation carried out with $n_{0}=2 n_{c}$ and $a_{0}=\sqrt{5}$ and for an electron temperature of $T_{e}=10 \mathrm{keV}$. Ions are fixed. As the electromagnetic wave penetrates inside the overdense plasma by SIT, new vortices are created and the propagation velocity of the wave front is close here to $v_{F}=0.09 c$. At time $t \omega_{p e}=861.3$, we clearly see the formation of ten vortices for the plasma length of $65.3 c / \omega_{p e}$, which corresponds to $10 \lambda_{e}$. The corresponding wave vector is then $k_{e} c / \omega_{p e}=0.960$ which is close to the expected value.

The case of a temperature of $T_{e}=200 \mathrm{keV}$ is shown in Fig. 10. The curve indicates an increase of the wave-front velocity $v_{F}$ reaching up to two times its initial value at low temperature (i.e., for $T_{e}=10 \mathrm{keV}$ ). However at $T_{e}=200 \mathrm{keV}$, the plasma exhibits a somewhat lower heating in the final state [here we have measured a value of the $\left\langle\gamma^{-1}\right\rangle$ factor close to 0.18]. In the context of a high value of the initial temperature, one may interpret this result as the effect of high Landau damping which may however slightly modify the EAW driven in a nonlinear way. Similar behavior was obtained at higher plasma temperature. We have found that now $\left\langle\gamma^{-1}\right\rangle$ is increasing slower as the temperature is bigger, in particular at $T_{e}=400 \mathrm{keV},\left\langle\gamma^{-1}\right\rangle$ is close to 0.20 , Landau damping might be however stronger if the nature of the plasma response was a Langmuir wave. It is the nature of the acoustic-like mode, characterized by trapping structure in
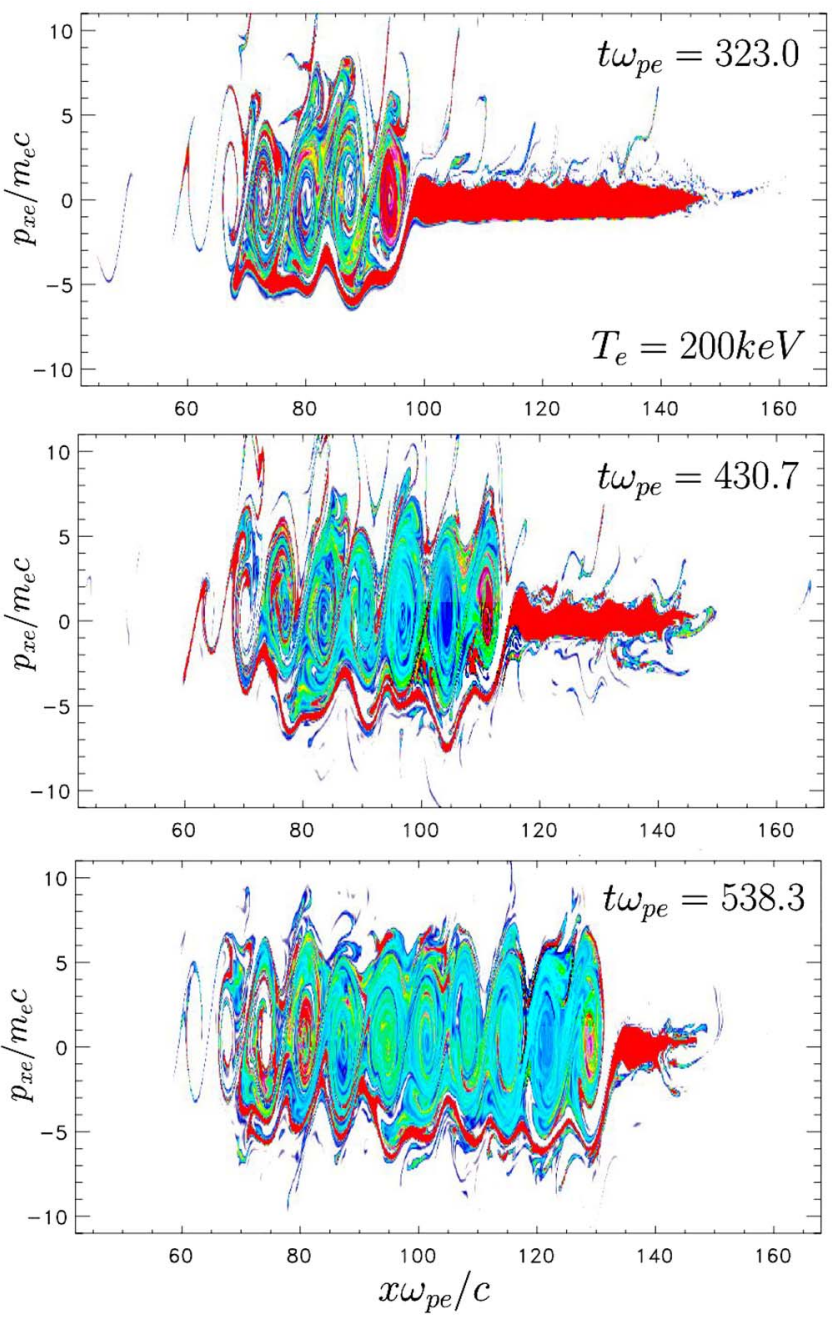

FIG. 10. (Color online) Phase space behavior of the electron distribution function for similar physical parameters $n_{0}=2 n_{c}$ and $a_{0}=\sqrt{5}$ (see Fig. 9) but for a higher value of the electron temperature of $T_{e}=200 \mathrm{keV}$. The case of a smaller value of the temperature was presented in Fig. 9. Here the progression of the wave front is faster indicating a higher value of its wave-front velocity. Ions are kept fixed.

phase space, which allows DBWH to be a significant process even if the plasma is initially hot.

It must be pointed out, that at a temperature of $T_{e}$ $=200 \mathrm{keV}$, the matching conditions are slightly modified. Indeed the parametric three-wave instability resonance requires here perfect matching both in frequency $\left(\omega_{0}=\omega_{r}+\omega_{e}\right.$, i.e., here $0.707=0.55+0.157$ in $\omega_{p e}$ units and in wave vectors $k_{0}=-k_{r}+k_{e}$, i.e., $0.55=-0.32+0.87$ in $\omega_{p e} c^{-1}$ units). From Eqs. (5) and (6), and using the fact that $\beta_{F}=0.18$ and $\beta_{0}$ $=1.285$, we take into account the Doppler shift, the values of $\omega_{r}=0.549 \omega_{p e}$ for the reflected wave frequency and $k_{r} c / \omega_{p e}$ $=0.322$ for the wave number, which are in good agreement with the numerical values, measured in spectra (not shown here).

We now choose to simulate DBWH at higher laser intensities. To demonstrate the influence of the ponderomotive force, a numerical simulation was performed with $n_{0} / n_{c}=2$ and a somewhat higher quiver momentum $a_{0}=3$, which corresponds to an intensity times $\lambda_{0}^{2}$ to $I \lambda_{0}^{2}=2.46$ 

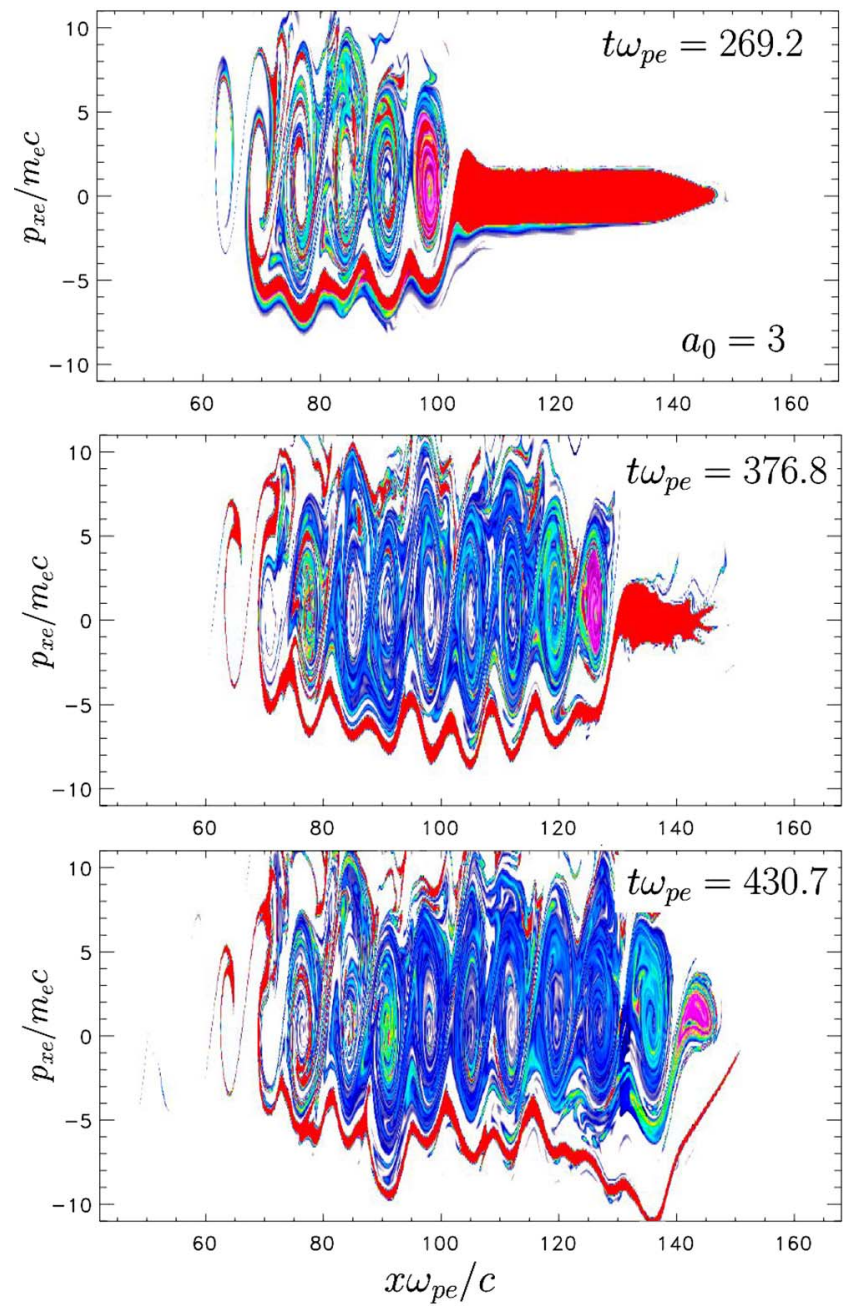

FIG. 11. (Color online) We now choose to simulate DBWH at higher laser intensities. The numerical simulation was performed with $n_{0} / n_{c}=2$ and a somewhat higher quiver momentum $a_{0}=3$. The electron temperature is kept to a value of $T_{e}=400 \mathrm{keV}$. Ions are fixed. The results must to be compared with those of Fig. 4 corresponding to a smaller value of the quiver momentum of $a_{0}=\sqrt{5}$. We found that strong trapping effects occur in phase space (the size in momentum of the vortices being bigger in comparison with that of the phase space vortices plotted in Fig. 4).

$\times 10^{19} \mathrm{~W} \mathrm{~cm}^{-2} \mu \mathrm{m}^{2}$. The temperature is kept to $T_{e}$ $=400 \mathrm{keV}$, the other physical parameters being identical to those used in Figs. 3 and 4.

Figure 11 displays the electron distribution function in phase space for $a_{0}=3$ (while in Fig. 4 the simulation was carried out for a somewhat smaller value of $a_{0}=\sqrt{5}$ ), showing the DBWH characteristically strong in nonlinear trapped particle modes. We found that strong trapping effects occur in phase space (the size in momentum of the vortices being bigger in comparison with that of the phase space vortices plotted in Fig. 4). Clearly these phase space structures move inside the plasma layer at velocity close to $v_{F}=0.245 \mathrm{c}$. The phase velocity is then easily and accurately measured as $v_{\varphi} / c=\omega_{e} / k_{e} c=0.24 / 0.915$ or $v_{\varphi}=0.26 c$ (i.e., $u_{\varphi}=p_{\varphi} / m_{e} c$ $=0.27$ ). This value is close to the wave front velocity. The analysis of electromagnetic spectra (not shown here) leads to a scattered light with a frequency $\omega_{r}=0.467 \omega_{p e}$ and the corresponding low-frequency electron mode is $\omega_{e}=\omega_{0}-\omega_{r}$
$=0.24 \omega_{p e}$ (the pump frequency being given by $\omega_{0}$ $\left.=0.707 \omega_{p e}\right)$. With $\left\langle\gamma^{-1}\right\rangle \simeq 0.125$, we find that the value of the pump frequency from the linear dispersion relation is $\omega_{0}$ $=\left(\omega_{p e}^{2}\left\langle\gamma^{-1}\right\rangle+k_{0}^{2} c^{2}\right)^{1 / 2} \simeq 0.705 \omega_{p e}$ using a value of the pump wave number of $k_{0} c / \omega_{p e}=0.61$. Corresponding matching conditions in wave numbers are then $k_{0}=-k_{r}+k_{e}$ with $k_{r} c / \omega_{p e}=0.305$ and $k_{e} c / \omega_{p e}=0.915$. Figure 11 shows the corresponding behavior of electron distribution function in phase space. From the bottom panel (plotted at time $t \omega_{p e}$ $=430$ ), we have estimated the wave vector $k_{e}$ of the electron mode to be $k_{e} c / \omega_{p e}=0.897$ (i.e., 9 wavelengths; the wavelength of this electron acoustic-like mode is thus close to $\lambda_{e} \simeq L_{\text {slab }} / 9=6.66 c / \omega_{p e}$ or equivalently $\left.k_{e} c / \omega_{p e} \simeq 0.94\right)$. On the other hand, Doppler-shift formulas (5) and (6) lead to an estimation of $\omega_{r}=0.479 \omega_{p e}$ for the reflected wave frequency and $k_{r} c / \omega_{p e}=0.319$ for the corresponding wave vector. These theoretical values were found in good agreement with the numerical values obtained in spectra.

\section{COUPLING WITH ELECTRON CAVITATION AND IAWS}

Competition between DBWH and electron cavitation is now investigated in this section. Fluid simulations show that an electron cavitation may arise and lead to the formation of a cavity in the plasma density filled by electromagnetic energy. The laser pulse, pushing electrons into the overdense plasma layer, creates a strongly overcritical plasma "wall." Part of the pulse is reflected back while a part of the energy pushes its way past the first wall and begins creating (in front of it) another plasma wall. As previously described in Ref. 3, the system then consists of a sequence of overcritical density spikes separated by deep density wells where parts of the electromagnetic radiation are trapped. We now focus on the creation of only one plasma "wall," i.e., one electron cavitation. Indeed Vlasov simulations show that this saturation mechanism thus seems to start out as hydrodynamical phenomenon but terminates with strongly nonlinear effects in which both ions and electrons participate. When the electron temperature is high enough, the Coulomb explosion takes place on the scale of the pump wavelength $\lambda_{0 V}$ and leads to the formation of electron cavities in plasma. Trapping of electromagnetic transverse fields inside the cavities is observed.

We choose to simulate a larger plasma slab. The first numerical simulation was performed with $n_{0} / n_{c}=1.6$ and a quiver momentum $a_{0}=\sqrt{2}$, which corresponds to an intensity times $\lambda_{0 V}^{2}$ to $I \lambda_{0 V}^{2} \simeq 5.45 \times 10^{18} \mathrm{~W} \mathrm{~cm}^{-2} \mu \mathrm{m}^{2}$. The plasma temperature is, respectively, chosen for electrons to $T_{e}$ $=100 \mathrm{keV}$ and for ions to $T_{i}=20 \mathrm{keV}$. We take again $m_{i}$ $=1836 m_{e}$. The physical system consists of a plasma slab of uniform density. The length of the slab is $L_{\text {plas }}=130 \mathrm{c} / \omega_{p e}$, which is close to $12 \lambda_{0}$ (we have taken $k_{0} c / \omega_{p e} \simeq 0.586$ ). The slab is again surrounded by a vacuum region of $L_{\mathrm{vac}}$ $=160 \mathrm{c} / \omega_{p e}$ on both sides and the jump of density on both sides is $L_{\text {jump }}=10 \mathrm{c} / \omega_{p e} \sim \lambda_{0 V}$ (pump wavelength in vacuum). The total number of grid points is $N_{x}=12800$ in the $x$ space and $N_{p_{x}}=513$ in both $p_{x e}$ and $p_{x i}$ momentum space. The pump frequency is then $\omega_{0}=0.790 \omega_{p e}$. Using $\left\langle\gamma^{-1}\right\rangle \simeq 0.28$, 

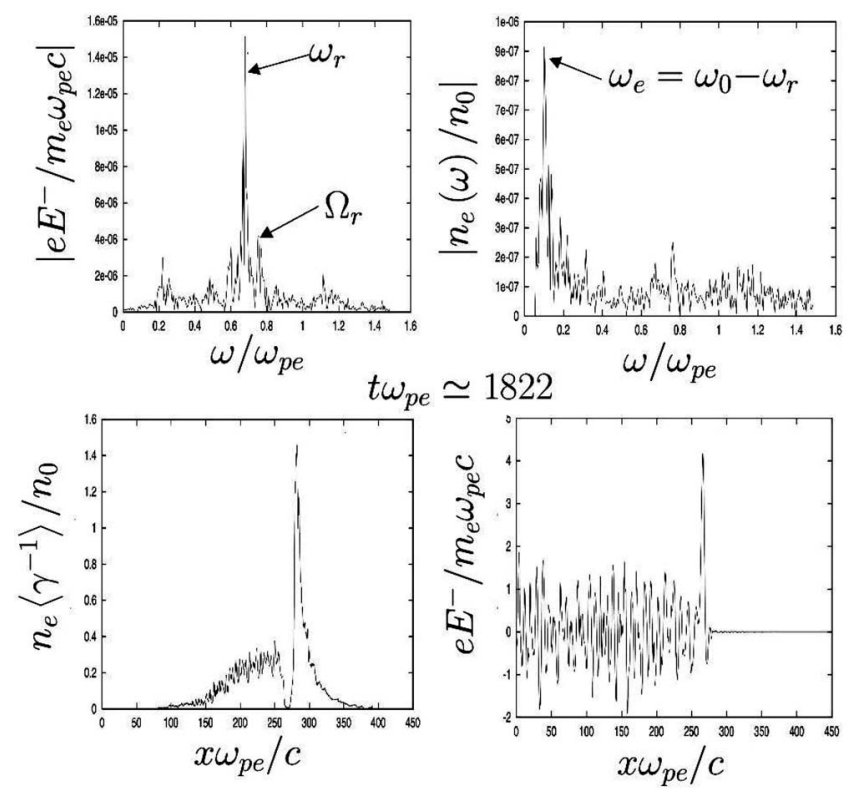

FIG. 12. Electromagnetic reflected (top left) and the electrostatic (top right panel) spectra. The electromagnetic spectrum exhibits a dominant peak at frequency of $\omega_{r}=0.68 \omega_{p e}$ in good agreement with the theoretical value $0.675 \omega_{p e}$ while the electrostatic spectrum shows that the dominant frequency is located near the value $\omega_{e}=0.11 \omega_{p e}$. Bottom panels: the relative electron density and its corresponding electromagnetic field $e E^{-} / m_{e} \omega_{p e} c$ at the same time $t \omega_{p e} \simeq 1822$. A cavity is rapidly formed and the electromagnetic radiation is trapped inside the density hole.

the value given by the linear dispersion relation of electromagnetic waves in plasma is $\omega_{0}=\left(\omega_{p e}^{2}\left\langle\gamma^{-1}\right\rangle+k_{0}^{2} c^{2}\right)^{1 / 2}$ $=0.789 \omega_{p e}$.

The corresponding three-wave parametric instability arises with the following matching conditions (1): $\omega_{0}=\omega_{r}$ $+\omega_{e}$ (here $0.790=0.675+0.115$ in $\omega_{p e}$ units) and $k_{0}=-k_{r}$ $+k_{e}$ (with $k_{0} c / \omega_{p e}=0.586$ and $k_{r} c / \omega_{p e}=0.42$ and $k_{e} c / \omega_{p e}$ $\simeq 1.006)$. As previously mentioned in Sec. II, $\left(\omega_{r},-k_{r}\right)$ satisfies the usual dispersion relation (2) and we have $\omega_{r}$ $=\left(\omega_{p e}^{2}\left\langle\gamma^{-1}\right\rangle+k_{r}^{2} c^{2}\right)^{1 / 2}=0.675 \omega_{p e}$ and the low-frequency acoustic-like electron wave is then driven by the beat wave process and obeys $\omega_{e} / \omega_{p e}=\left(k_{e} c / \omega_{p e}\right)\left(v_{F} / c\right)=1.006 \times 0.115$ $\simeq 0.12$. From Eqs. (5) and (6) we obtain $\omega_{r}=0.675 \omega_{p e}$ and $k_{r}=0.417 \omega_{p e} / c$ in good agreement with values observed in simulation.

Figure 12 (top panels) illustrates these results: we have plotted the electromagnetic reflected (top left) and the electrostatic (top right panel) spectra. The electromagnetic spectrum exhibits a dominant peak at frequency $\omega_{r}=0.68 \omega_{p e}$ in good agreement with the theoretical value while the electrostatic spectrum shows that the dominant frequency is located near the value $\omega_{e}=0.11 \omega_{p e}$. We have also represented in Fig. 12 (bottom panels) the relative electron density and its corresponding electromagnetic field $e E^{-} / m_{e} \omega_{p e} c$ at the same time $t \omega_{p e} \simeq 1822$. A cavity is rapidly formed and the electromagnetic radiation is trapped inside the density hole, which on the long time becomes quasineutral. Thus we first confirm numerical results obtained in Ref. 3.

The behavior of the distribution function is shown in Fig. 13 at three different times during the simulation. The top panel of Fig. 13 clearly shows the beginning of the electron
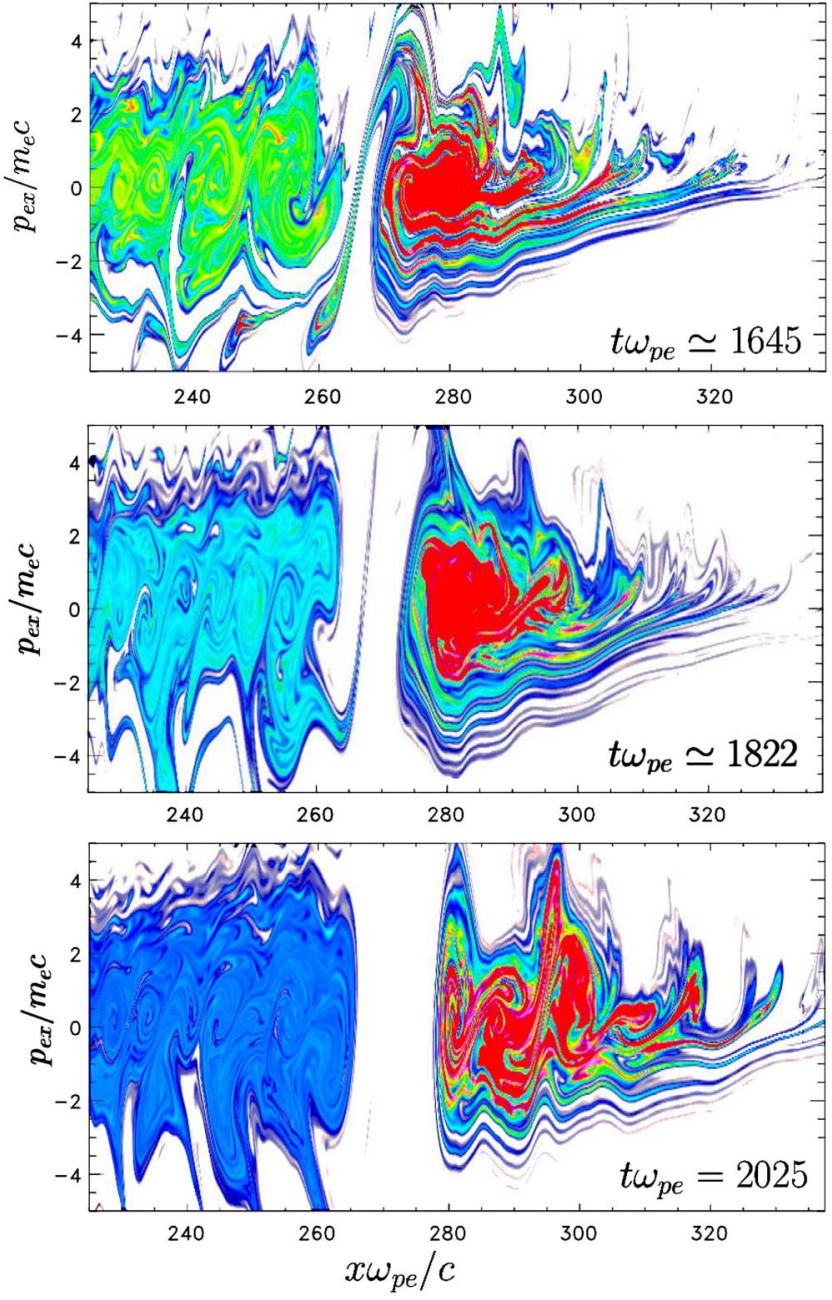

FIG. 13. (Color online) Behavior of the electron distribution function at three different times showing the formation of the electron cavitation. The numerical simulation was performed with $n_{0} / n_{c}=1.6$ and a quiver momentum $a_{0}=\sqrt{2}$. To take into account strong charge separation, ions are now mobile with $m_{i}=1836 m_{e}$. The physical system consists of a plasma slab of uniform density. The length of the slab is $L_{\text {plas }}=130 \mathrm{c} / \omega_{p e}$, which is close to $12 \lambda_{0}$.

cavitation at time $t \omega_{p e}=1645$. The density cavity is formed which cuts the plasma into two regions at time $t \omega_{p e}=1822$. Whereas the Coulomb explosion takes place, ion and electron density peaks reach up to $1.8 n_{c}$ making the region overdense for the incoming electromagnetic wave. The strong increase of the ion density making the penetration impossible of the intense laser pulse is due to the wave breaking of the ion distribution function. The information obtained in real space and of the electron dynamics is supplemented by looking at the ion dynamics in phase space presented in Fig. 14 (see the top panel). In particular the progression of the electromagnetic wave is stopped due to the strong increase of the ion (and electron) density near the wave front making the plasma overdense for the incident laser pulse. A wave breaking is clearly discernible in space located near $x \omega_{p e} / c=290$. Similar behavior was recently observed in a somewhat different regime of the laser-plasma interaction and in particular in stimulated Brillouin scattering (see, for more details, Ref. 14). 

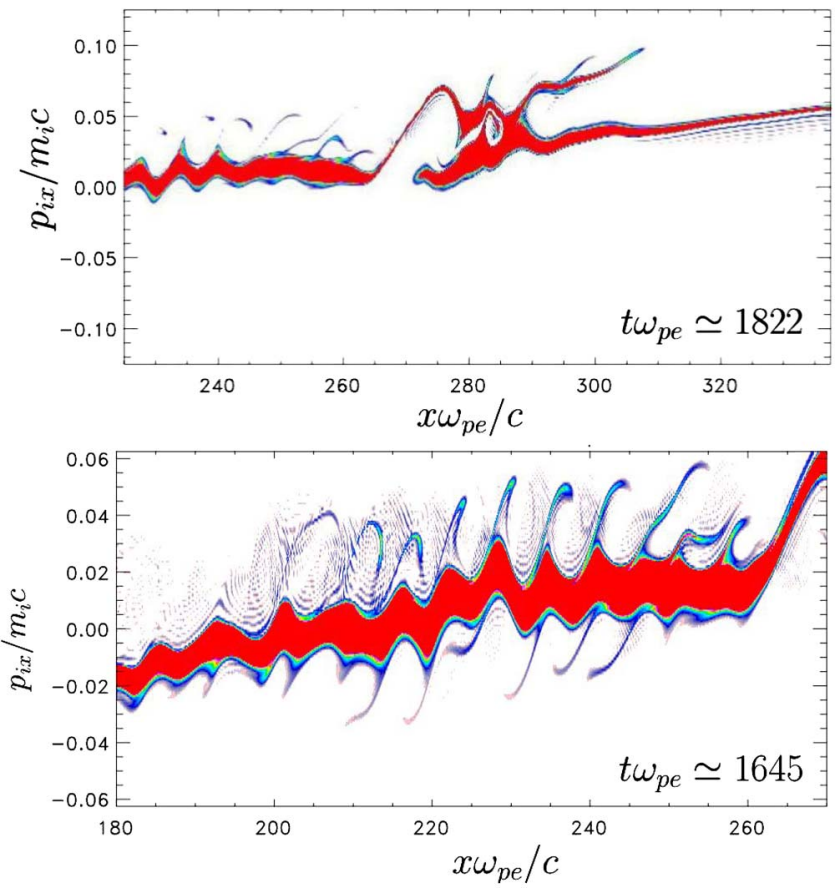

FIG. 14. (Color online) Corresponding ion distribution function representation in phase space showing the ion wave-breaking located at around the position $x \omega_{p e} / c=290$ at time $t \omega_{p e} \simeq 1822$ in the top panel. Bottom panel: at the beginning of the electron cavitation the wave front velocity is weak enough to excite IAW. At time $t \omega_{p e} \simeq 1645$ we make a zoom of the ion distribution function just in front of the cavitation region. We may observe the beginning of trapping.

The presence of an ion wave breaking can be identified in the ion phase space, i.e., in the top panel of Fig. 14 at time $t \omega_{p e}=1822$. The corresponding electron phase space is plotted in the middle panel in Fig. 13. From our Vlasov simulation data, the interaction of the laser pulse with the overdense plasma slab was identified as a two-step mechanism. First DBWH is the first process occurring in time in the simulation. As a result the wave front propagation is fast with a value of the velocity $v_{F}$ close to $0.12 c$. This result is similar to that presented in previous sections and is not shown here. Then this propagation is strongly modified in the second phase of the interaction characterized by the electron cavitation formation and the laser pulse deceleration. We have examined this kind of behavior in detail: the wave front velocity $v_{F}$ was found to be very close to the value $v_{F} \simeq 0.025 c$, while an analytical prediction based upon the formula (7) gives $v_{F} \simeq 0.026 c$. This behavior may be clearly observed in Fig. 13. This means that the beat wave process between the incoming pump and the reflected wave is maintained even in this regime where the velocity of the wave front is weak. There is, however, an important modification to the original picture, in which electron acoustic modes are driven. It appears that now for smaller wave front velocities, it is IAWs which are excited and growing in a similar way. Matching conditions (1) are now replaced by

$$
\omega_{0}=\Omega_{r}+\Omega_{i} \text { and } k_{0}=-K_{r}+K_{i} .
$$

Here we have $\omega_{0}=0.790 \omega_{p e}$ for the pump, $\Omega_{r}$ $=0.760 \omega_{p e}$ for the reflected electromagnetic wave, and $\Omega_{i}$
$=0.03 \omega_{p e}$ for the IAW (the corresponding values for the wave numbers are then $k_{0}=0.586 \omega_{p e} / c, K_{r}=0.545 \omega_{p e} / c$, and $\left.K_{i}=1.13 \omega_{p e} / c\right)$. Again the reflected electromagnetic wave obeys the conditions (5) and (6) obtained by taking into account the relativistic Doppler shift. Using formulas (5) and (6) we obtain $\Omega_{r}=0.761 \omega_{p e}$ and $K_{r}=0.547 \omega_{p e} / c$ which are in good agreement with the numerical values. Here $\left(\Omega_{i}, K_{i}\right)$ satisfies (more and less) the linear dispersion relation of the IAW, i.e., $\Omega_{i}=K_{i} c_{s} \sim K_{i} v_{F}$ or $1.13 \omega_{p e} / c \times 0.025 c=0.03 \omega_{p e}$. In this DBWH regime, the plasma has apparently now become hot enough to support IAW of resonance phase velocity (i.e., $v_{\varphi}=\Omega_{i} / K_{i} \sim c_{s}$ with $c_{s}=v_{\text {th }} \sqrt{m_{e} / m_{i}} \sim c \sqrt{m_{e} / m_{i}}$ ). The ion mode enters in a nonlinear regime characterized by trapping structures. This feature is also observed in the ion phase space in the bottom panel in Fig. 14 which exhibits the beginning of the trapping of the ion mode with a wavelength of $\lambda_{i}=6.46$ or equivalently $K_{i} c / \omega_{p e}=0.97$ which is close to the expected value of $K_{i} c / \omega_{p e}=1.13$ predicted in the DBWH scenario. The figure, plotted at time $t \omega_{p e}=1645$, i.e., at the beginning of the electron cavitation shows a zoom of the ion distribution function in phase space in a region located in front of the cavitation.

\section{CONCLUSION AND DISCUSSION}

To conclude, we have considered the interaction of a strong electromagnetic wave with moderately overdense plasma in 1D geometry in normal incidence under conditions that allow for self-induced transparency. We have review here the main phases which characterize the relativistic regime of interaction, as resulting from our Vlasov-Maxwell simulations.

During the propagation of the laser pulse, a relativistic Doppler shift takes place at the moving wave front and causes the beating of the incoming pump wave with its reflected Doppler-shifted wave. The resulting underdense plasma, located in front of the wave front, is now subject to a new parametric excitation which is able to drive and maintain a low-frequency electron mode. A Doppler-beat-wave heating is then produced leading to the generation of electron acoustic-like waves driven at a nonlinear level.

In systematic simulation studies of this scheme, we varied the characteristics of the DBWH instability: the ratio of the electron density to the critical density until a limit value for which the propagation is not possible, the electron plasma temperature and finally the intensity of the incident laser pulse. Our investigation reveals that it is the beating of the pump with the reflected wave which determines the resonance condition and the characteristics of the low-frequency EAW-like wave. For a large range of physical parameters we have demonstrated the possibility of strong growth of this new beat wave heating process.

At the beginning of the DBWH mechanism, the beat wave drives an EAW; there is however a change in the nature of this initially EAW, which is no longer a simple acoustic mode, but as we hypothesize a more complex structure characterized by trapping effects in the form of phase space ("bumps") structures (see also Ref. 18). This brings us to an important question of the physical origin of these driven 
electron modes. These modes seem to differ in trapped electron acoustic waves (TEAW), recently observed in the Trident experiment in Ref. 19 and linked to the theoretical works of Rose et al. presented in Ref. 20. In Ref. 20 the nonlinear dispersion relation obtained in this model is qualitatively different from the one usually prescribed by Landau's analysis of the linearized Vlasov equation which leads to the well-known Bohm-Gross modes or Langmuir waves. A second lower acoustic branch was obtained corresponding to the TEAW, whose phase velocity is close to $1.3 v_{\text {th }}$, a variant version of the well-known Bernstein-Greene-Kruskal (BGK) equilibria in Ref. 16. Recent work using Vlasov simulations (as presented in Refs. 22 and 23) have shown the importance of trapping effects in such experiments at moderate laser intensities.

The situation is however somewhat more complex in the regime where the driving lasers are at relativistic intensities as met in the SIT scenario. Excitations of a second wave have been observed. We stress that the plasma response here is not just a simple driven mode (occurring initially in the form of an EAW in the linear case) but a more complicated state corresponding to the mixing of the forced EAW until a nonlinear regime and a KEEN wave excited at the same wave number. DBWH preheats the plasma and acts as a seed to excite the KEEN modes since the plasma is now hot enough to create these modes. KEEN waves in this frequency and phase velocity range can be produced both if the plasma is initially hot enough and if coherently driven up to self-sustaining levels.

Once the plasma layer crossing by the front wave is achieved, DBWH ceases. As the result of the primary DBWH, the plasma became relativistically hot and underdense in the region where the beat wave is effective and this may support the formation of self-sustained electron nonlinear waves. The backscattered wave was found to be driven near critical, i.e., $\omega_{s} \simeq \omega_{p e}\left\langle\gamma^{-1}\right\rangle^{1 / 2}$ that means $k_{s} \simeq 0$ as predicted in Ref. 15. The explanation of this behavior (at least for relativistically strong field and relativistically hot plasmas) is that there is a "radiating pseudocavity" electromagnetic mode with relatively low loss for the plasma slab at a frequency close to $\omega_{p e}\left\langle\gamma^{-1}\right\rangle^{1 / 2}$ and this allows the initial excitation of the BGK-type ${ }^{16}$ waves at a well-defined frequency $\omega_{0}-\omega_{s}$.

The physical interpretation of the observed anomalous heating met in the SIT scenario, where information seems to be linked to EAW-like modes requires careful analysis at more than one level. It will become apparent that nonlinear effects and in particular trapping effects must be brought in to fully understand the implications of SIT. In particular, the hydrodynamical approach is usually justified only for systems near the thermodynamic equilibrium. In nonequilibrium systems, one, in principle, has to apply some suitable kinetic models. The question of whether the hydrodynamical approach involves substantially more uncertainties than we would expect from the kinetic description is therefore of fundamental importance and closely related to the question of whether current fluid models are adequate to study SIT.
Vlasov simulations provide a unique opportunity to adequately describe the nonlinear particle dynamics in detail, including particle trapping. Obtaining this insight into the very fine description of the distribution function was only possible with the Vlasov model which can provide a great deal of resolution in phase space.

\section{ACKNOWLEDGMENTS}

The authors are indebted to the IDRIS center (Orsay France) for computer time allocation on the NEC-SX5 and IBM-Power4 computers. The authors would like to acknowledge P. Bertrand, T. W. Johnston, and W. Tikhonchuck for fruitful discussions on the topics.

${ }^{1}$ M. Tabak, J. Hammer, M. E. Glinsky, W. L. Kruer, S. C. Wilks, J. Woodworth, E. M. Campbell, M. D. Perry, and R. J. Mason, Phys. Plasmas 1, 1626 (1994).

${ }^{2}$ M. Tushentsov, A. Kim, F. Cattani, D. Anderson, and M. Lisak, Phys. Rev. Lett. 87, 275002 (2001); see also F. Cattani, A. Kim, D. Anderson, and M. Lisak, Phys. Rev. E 62, 1234 (2000).

${ }^{3}$ V. I. Berezhiani, D. P. Gacuchava, S. V. Mikeladze, K. I. Sigua, N. L. Tsintdadze, S. M. Mahajan, Y. Kishimoto, and K. Nishikawa, Phys. Plasmas 12, 062308 (2005)

${ }^{4}$ J. Denavit, Phys. Rev. Lett. 69, 3052 (1992); see also A. Macchi, F. Cattani, T. V. Liseykina, and F. Cornolti, ibid. 94, 165003 (2005).

${ }^{5}$ S. Guérin, P. Mora, J. C. Adam, A. Héron, and G. Laval, Phys. Plasmas 3, 2693 (1996).

${ }^{6}$ V. A. Vshiutov, N. M. Naumova, F. Pegoraro, and S. V. Bulanov, Phys. Plasmas 5, 2727 (1998)

${ }^{7}$ Q. L. Dong, Z. M. Sheng, M. Y. Yu, and J. Zhang, Phys. Rev. E 68, 026408 (2003).

${ }^{8}$ Y. Sentoku, T. E. Cowan, A. Kemp, and H. Ruhl, Phys. Plasmas 10, 2009 (2003).

${ }^{9}$ P. Bertrand, A. Ghizzo, T. W. Johnston, E. Fijalkow, and M. R. Feix, Phys. Fluids B 2, 1028 (1990); see also T. W. Johnston, P. Bertrand, A. Ghizzo, M. Shoucri, E. Fijalkow, and M. R. Feix, ibid. 4, 2523 (1992); A. Ghizzo, P. Bertrand, J. Lebas, T. W. Johnston, and M. Shoucri, Phys. Plasmas 5, 4041 (1998).

${ }^{10}$ P. Bertrand, M. Albrecht-Marc, T. Reveille, and A. Ghizzo, Transp. Theory Stat. Phys. 34, 103 (2005).

${ }^{11}$ F. Huot, A. Ghizzo, P. Bertrand, E. Sonnendrucker, and O. Coulaud, J. Comput. Phys. 185, 512 (2003).

${ }^{12}$ B. I. Cohen, A. N. Kaufman, and K. M. Watson, Phys. Rev. Lett. 29, 581 (1972).

${ }^{13}$ T. Tajima and J. M. Dawson, Phys. Rev. Lett. 43, 267 (1979).

${ }^{14}$ S. Weber, C. Riconda, and V. T. Tikhonchuk, Phys. Plasmas 12, 043101 (2005).

${ }^{15}$ A. Ghizzo, T. W. Johnston, T. Reveille, P. Bertrand, and M. AlbrechtMarc, Phys. Rev. E 74, 046407 (2006).

${ }^{16}$ I. B. Bernstein, J. M. Greene, and M. D. Kruskal, Phys. Rev. 108, 507 (1957).

${ }^{17}$ L. Nikolic, M. M. Skoric, S. Ishiguro, and T. Sato, Phys. Rev. E 66, 036404 (2002).

${ }^{18}$ M. Albrecht-Marc, A. Ghizzo, T. Reveille, P. Bertrand, and T. W. Johnston, Transp. Theory Stat. Phys. 34, 127 (2005).

${ }^{19}$ D. S. Montgomery, R. J. Focia, H. A. Rose, D. A. Russell, J. A. Cobble, J. C. Fernandez, and R. P. Johnson, Phys. Rev. Lett. 87, 155001 (2001); D. S. Montgomery, J. A. Cobble, J. C. Fernandez, R. J. Focia, R. P. Johnson, N. Renard-LeGalloudec, H. A. Rose, and D. A. Russell, Phys. Plasmas 9, 2311 (2002).

${ }^{20}$ H. A. Rose and D. A. Russell, Phys. Plasmas 8, 4784 (2001).

${ }^{21}$ B. B. Afeyan, K. Won, V. Savchenko, T. W. Johnston, A. Ghizzo, and P. Bertrand, Third International Conference on Inertial Fusion Sciences and Applications MO34, Monterey, California, 7-12 September 2003.

${ }^{22}$ N. J. Sircombe, T. D. Arber, and R. O. Dendy, Plasma Phys. Controlled Fusion 48, 1141 (2006).

${ }^{23}$ D. J. Strozzi, E. A. Williams, A. B. Langdon, and A. Bers, Phys. Plasmas 14, 013104 (2007). 